Supporting information for

\title{
Palladium-catalyzed Cross-Coupling of Aryl Electrophiles with Dimethylalkynylaluminum reagents.
}

Baomin Wang, Martine Bonin* and Laurent Micouin.*

Laboratoire de Chimie Thérapeutique, UMR 8638 associée au CNRS et à l'Université René Descartes, Faculté des Sciences Pharmaceutiques et Biologiques, 4 av de l'Observatoire, 75270 Paris cedex 06 (France) laurent.micouin@univ-paris5.fr

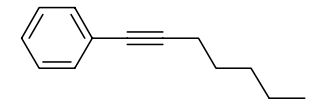

Hept-1-ynyl-benzene ${ }^{1}$

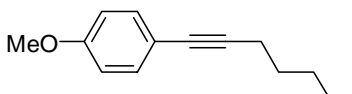

Hept-1-ynyl-4-methoxy-benzene ${ }^{2}$

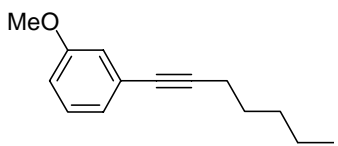

Hept-1-ynyl-3-methoxy-benzene ${ }^{3}$

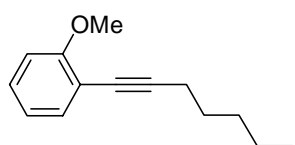

Hept-1-ynyl-2-methoxy-benzene ${ }^{2}$

$\mathrm{HO}-$

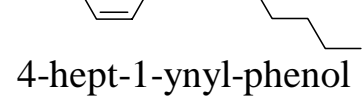

${ }^{1} \mathrm{H}$ NMR $\left(400 \mathrm{MHz}, \mathrm{CDCl}_{3}\right) \delta 7.41(\mathrm{~m}, 2 \mathrm{H}), 7.27(\mathrm{~m}$, $3 \mathrm{H}), 2.42(\mathrm{t}, J=7.1 \mathrm{~Hz}, 2 \mathrm{H}), 1.62(\mathrm{~m}, 2 \mathrm{H}), 1.34-1.48$ $(\mathrm{m}, 4 \mathrm{H}), 0.93(\mathrm{t}, J=7.2 \mathrm{~Hz}, 3 \mathrm{H})$.

${ }^{1} \mathrm{H}$ NMR (400 MHz, $\left.\mathrm{CDCl}_{3}\right) \delta 7.53(\mathrm{~d}, J=7.4 \mathrm{~Hz}$, $2 \mathrm{H}), 6.82(\mathrm{~d}, J=7.4 \mathrm{~Hz}, 2 \mathrm{H}), 3.80(\mathrm{~s}, 3 \mathrm{H}), 2.40(\mathrm{t}, J=$ $7.1 \mathrm{~Hz}, 2 \mathrm{H}), 1.61(\mathrm{~m}, 2 \mathrm{H}), 1.34-1.48(\mathrm{~m}, 4 \mathrm{H}), 0.93(\mathrm{t}$, $J=7.2 \mathrm{~Hz}, 3 \mathrm{H})$.

${ }^{1} \mathrm{H}$ NMR (400 MHz, $\left.\mathrm{CDCl}_{3}\right) \delta 7.19(\mathrm{t}, J=7.9 \mathrm{~Hz}$, $1 \mathrm{H}), 7.00(\mathrm{~d}, J=7.6 \mathrm{~Hz}, 1 \mathrm{H}), 6.94(\mathrm{~m}, 1 \mathrm{H}), 6.82(\mathrm{ddd}$, $J=8.4,2.6,0.8 \mathrm{~Hz}, 1 \mathrm{H}), 3.80(\mathrm{~s}, 3 \mathrm{H}), 2.40(\mathrm{t}, J=7.1$ $\mathrm{Hz}, 2 \mathrm{H}), 1.61(\mathrm{~m}, 2 \mathrm{H}), 1.34-1.48(\mathrm{~m}, 4 \mathrm{H}), 0.93$ (t, $J=$ $7.2 \mathrm{~Hz}, 3 \mathrm{H})$.

${ }^{1} \mathrm{H}$ NMR $\left(400 \mathrm{MHz}, \mathrm{CDCl}_{3}\right) \delta 7.39(\mathrm{dd}, J=7.5,1.6$ $\mathrm{Hz}, 1 \mathrm{H}), 7.24(\mathrm{td}, J=7.5,1.6 \mathrm{~Hz}, 1 \mathrm{H}), 6.88(\mathrm{~m}, 2 \mathrm{H})$, $3.88(\mathrm{~s}, 3 \mathrm{H}), 2.47(\mathrm{t}, J=7.1 \mathrm{~Hz}, 2 \mathrm{H}), 1.62(\mathrm{~m}, 2 \mathrm{H})$, $1.34-1.48(\mathrm{~m}, 4 \mathrm{H}), 0.93(\mathrm{t}, J=7.2 \mathrm{~Hz}, 3 \mathrm{H})$.

Air sensitive oil. ${ }^{1} \mathrm{H}$ NMR (400 MHz, $\left.\mathrm{CDCl}_{3}\right) \delta 7.28$ $(\mathrm{d}, J=8.6 \mathrm{~Hz}, 2 \mathrm{H}), 6.75(\mathrm{~d}, J=8.6 \mathrm{~Hz}, 2 \mathrm{H}), 4.96(\mathrm{br}$ $\mathrm{s}, 1 \mathrm{H}), 2.39(\mathrm{t}, J=7.1 \mathrm{~Hz}, 2 \mathrm{H}), 1.61(\mathrm{~m}, 2 \mathrm{H}), 1.34-$ $1.48(\mathrm{~m}, 4 \mathrm{H}), 0.93(\mathrm{t}, J=7.2 \mathrm{~Hz}, 3 \mathrm{H}) .{ }^{13} \mathrm{C}$ NMR $(100$ $\left.\mathrm{MHz}, \mathrm{CDCl}_{3}\right) \delta 155.0,133.2,116.6,115.4,89.1,80.3$, 31.2, 28.7, 22.4, 19.4, 14.13 ; IR $\left(\mathrm{cm}^{-1}\right)$ 3394, 2956, 2931, 2859, 1608. 

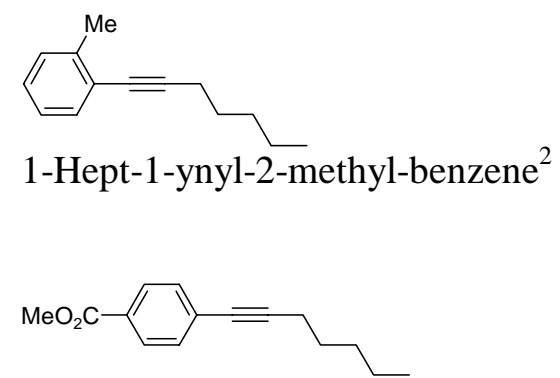

4-Hept-1-ynyl-benzoic acid methyl ester $^{4}$
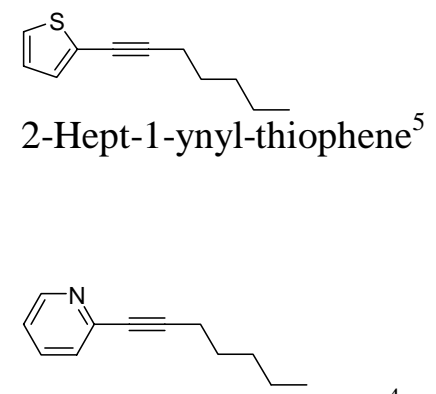

2-Hept-1-ynyl-pyridine ${ }^{4}$

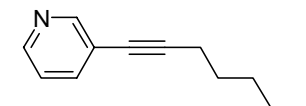

3-Hept-1-ynyl-pyridine

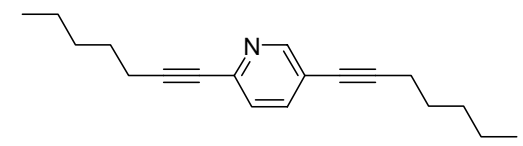

2,5-Di-hept-1-ynyl-pyridine
${ }^{1} \mathrm{H}$ NMR (400 MHz, $\left.\mathrm{CDCl}_{3}\right) \delta 7.37(\mathrm{~d}, J=7.3 \mathrm{~Hz}$, $1 \mathrm{H}), 7.08-7.17(\mathrm{~m}, 3 \mathrm{H}), 2.44(\mathrm{t}, J=7.1 \mathrm{~Hz}, 2 \mathrm{H}), 1.62$ (m, 2H), 1.34-1.48 (m, 4H), 0.93 (t, $J=7.2 \mathrm{~Hz}, 3 \mathrm{H})$.

${ }^{1} \mathrm{H}$ NMR (400 MHz, $\left.\mathrm{CDCl}_{3}\right) \delta 7.95(\mathrm{~d}, J=8.1 \mathrm{~Hz}$, $2 \mathrm{H}), 7.44(\mathrm{~d}, J=8.1 \mathrm{~Hz}, 2 \mathrm{H}), 3.91(\mathrm{~s}, 3 \mathrm{H}), 2.43(\mathrm{t}, J=$ $7.1 \mathrm{~Hz}, 2 \mathrm{H}), 1.61(\mathrm{~m}, 2 \mathrm{H}), 1.34-1.48(\mathrm{~m}, 4 \mathrm{H}), 0.93(\mathrm{t}$, $J=7.2 \mathrm{~Hz}, 3 \mathrm{H})$.

${ }^{1} \mathrm{H}$ NMR (400 MHz, $\left.\mathrm{CDCl}_{3}\right) \delta 7.17(\mathrm{dd}, J=5.2,1.0$ $\mathrm{Hz}, 1 \mathrm{H}), 7.12(\mathrm{dd}, J=3.5,0.8 \mathrm{~Hz}, 1 \mathrm{H}), 6.94(\mathrm{dd}, J=$ $5.2,3.5 \mathrm{~Hz}, 1 \mathrm{H}), 2.43(\mathrm{t}, J=7.1 \mathrm{~Hz}, 2 \mathrm{H}), 1.61(\mathrm{~m}$, 2H), 1.34-1.48 (m, 4H), 0.93 (t, $J=7.2 \mathrm{~Hz}, 3 \mathrm{H})$.

${ }^{1} \mathrm{H}$ NMR (400 MHz, $\left.\mathrm{CDCl}_{3}\right) \delta 8.53(\mathrm{~d}, J=4.1 \mathrm{~Hz}$, $1 \mathrm{H}), 7.59(\mathrm{td}, J=7.8,1.8 \mathrm{~Hz}, 1 \mathrm{H}), 7.35(\mathrm{~d}, J=7.8 \mathrm{~Hz}$, 1H), 7.16 (ddd, $J=7.8,4.1,1.8 \mathrm{~Hz}, 1 \mathrm{H}), 2.43$ (t, $J=$ $7.1 \mathrm{~Hz}, 2 \mathrm{H}), 1.61(\mathrm{~m}, 2 \mathrm{H}), 1.34-1.48(\mathrm{~m}, 4 \mathrm{H}), 0.91(\mathrm{t}$, $J=7.2 \mathrm{~Hz}, 3 \mathrm{H})$.

Oil. ${ }^{1} \mathrm{H}$ NMR (400 MHz, $\left.\mathrm{CDCl}_{3}\right) \delta 8.62$ (br s, $1 \mathrm{H}$ ), $8.47(\mathrm{~d}, J=4.9 \mathrm{~Hz}, 1 \mathrm{H}), 7.65(\mathrm{~d}, J=7.8 \mathrm{~Hz}, 1 \mathrm{H})$, $7.16(\mathrm{dd}, J=7.8,4.8 \mathrm{~Hz}, 1 \mathrm{H}), 2.42(\mathrm{t}, J=7.1 \mathrm{~Hz}$, 2H), $1.61(\mathrm{~m}, 2 \mathrm{H}), 1.34-1.48(\mathrm{~m}, 4 \mathrm{H}), 0.92(\mathrm{t}, J=7.2$ $\mathrm{Hz}, 3 \mathrm{H}) ;{ }^{13} \mathrm{C}$ NMR $\left(100 \mathrm{MHz}, \mathrm{CDCl}_{3}\right) \delta 152.4$, 147.9, 138.4, 122.9, 121.3, 94.2, 77.5, 31.2, 28.3, 22.3, 19.5, 14.1; IR $\left(\mathrm{cm}^{-1}\right)$ 3029, 2956, 2931, 2859, 2241, 2221, 1560, 1475, 1406 ; HRMS (ES+) for $\mathrm{C}_{12} \mathrm{H}_{16} \mathrm{~N}$ : cald.: 174.1283 , found: 174.1279 .

Oil. ${ }^{1} \mathrm{H}$ NMR (400 MHz, $\left.\mathrm{CDCl}_{3}\right) \delta 8.54(\mathrm{~d}, J=1.3$ $\mathrm{Hz}, 1 \mathrm{H}), 7.58(\mathrm{dd}, J=8.1,2.1 \mathrm{~Hz}, 1 \mathrm{H}), 7.27(\mathrm{~d}, J=$ $8.1 \mathrm{~Hz}, 1 \mathrm{H}), 2.43(\mathrm{~m}, 4 \mathrm{H}), 1.63(\mathrm{~m}, 4 \mathrm{H}), 1.34-1.48$ $(\mathrm{m}, 8 \mathrm{H}), 0.92(\mathrm{~m}, 6 \mathrm{H}) ;{ }^{13} \mathrm{C}$ NMR $\left(100 \mathrm{MHz}, \mathrm{CDCl}_{3}\right)$ $\delta$ 152.4, 142.0, 138.4, 126.0, 119.7, 95.5, 92.7, 80.3, $77.5,31.2,28.3,28.1,22.3,19.6,19.5,14.0$; IR $\left(\mathrm{cm}^{-}\right.$ 1) 2931, 2860, 2225, 1468, 1363 ; HRMS (ES+) for $\mathrm{C}_{12} \mathrm{H}_{16} \mathrm{~N}$ : cald.: 268.2065, found: 268.2053. 


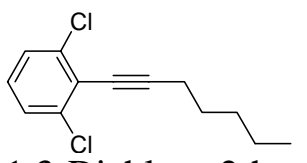

1,3-Dichloro-2-hept-1-ynylbenzene

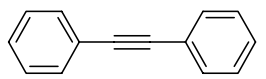

Diphenylacetylene ${ }^{6}$

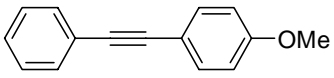

1-Methoxy-4-phenethynyl-benzene ${ }^{7}$

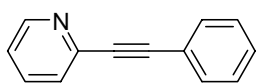

2-(4-Methoxy-phenethynyl)pyridine $^{8}$

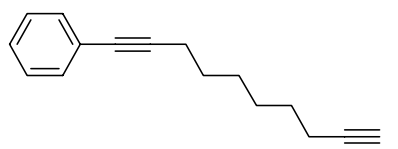

Deca-1,9-diynyl-benzene

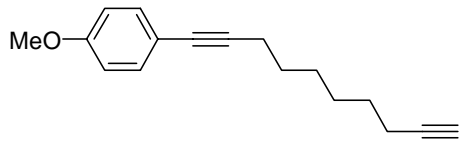

Deca-1,9-diynyl-4-methoxybenzene
Oil. ${ }^{1} \mathrm{H}$ NMR $\left(400 \mathrm{MHz}, \mathrm{CDCl}_{3}\right) \delta 7.29(\mathrm{t}, J=8.1$ $\mathrm{Hz}, 2 \mathrm{H}), 7.11(\mathrm{t}, J=8.1 \mathrm{~Hz}, 1 \mathrm{H}), 2.54(\mathrm{t}, J=7.0 \mathrm{~Hz}$, $2 \mathrm{H}), 1.68(\mathrm{~m}, 2 \mathrm{H}), 1.47-1.55(\mathrm{~m}, 2 \mathrm{H}), 1.35-1.40(\mathrm{~m}$, $2 \mathrm{H}), 0.93(\mathrm{t}, J=7.2 \mathrm{~Hz}, 3 \mathrm{H}) ;{ }^{13} \mathrm{C}$ NMR $(100 \mathrm{MHz}$, $\left.\mathrm{CDCl}_{3}\right) \delta 137.2,128.2,127.4,124.0,102.3,77.4$, 75.13, 28.2, 22.3, 19.9, 14.1; IR $\left(\mathrm{cm}^{-1}\right)$ 2931, 2859, 2240, 1553, 1446, 1428, 789, 774, 720 .

${ }^{1} \mathrm{H}$ NMR $\left(400 \mathrm{MHz}, \mathrm{CDCl}_{3}\right) \delta 7.56(\mathrm{~m}, 4 \mathrm{H}), 7.39(\mathrm{~m}$, $6 \mathrm{H})$.

${ }^{1} \mathrm{H}$ NMR (400 MHz, $\left.\mathrm{CDCl}_{3}\right) \delta$ 7.47-7.53 (m, 4H), 7.27-7.35 (m, 3H), $6.87(\mathrm{~d}, J=8.8 \mathrm{~Hz}, 2 \mathrm{H}), 3.84(\mathrm{~s}$, $3 \mathrm{H})$.

${ }^{1} \mathrm{H}$ NMR $\left(400 \mathrm{MHz}, \mathrm{CDCl}_{3}\right) \delta 8.62(\mathrm{~d}, J=4.8 \mathrm{~Hz}$, $1 \mathrm{H}), 7.67(\mathrm{td}, J=7.7,1.8 \mathrm{~Hz}, 1 \mathrm{H}), 7.60(\mathrm{~m}, 2 \mathrm{H}), 7.52$ $(\mathrm{dd}, J=7.8,0.8 \mathrm{~Hz}, 1 \mathrm{H}) 7.36(\mathrm{~m}, 3 \mathrm{H}), 7.24(\mathrm{~m}, 1 \mathrm{H})$.

Oil. ${ }^{1} \mathrm{H}$ NMR (400 MHz, $\left.\mathrm{CDCl}_{3}\right) \delta 7.40(\mathrm{~m}, 2 \mathrm{H})$; $7.28(\mathrm{~m}, 3 \mathrm{H}) ; 2.42(\mathrm{t}, J=7.0 \mathrm{~Hz}, 2 \mathrm{H}), 2.21(\mathrm{td}, J=$ $6.9,2.5 \mathrm{~Hz}, 2 \mathrm{H}), 1.95(\mathrm{t}, J=2.5 \mathrm{~Hz}, 1 \mathrm{H}), 1.45-1.63$ $\mathrm{m}, 8 \mathrm{H}) . \delta{ }^{13} \mathrm{C}$ NMR $\left(100 \mathrm{MHz}, \mathrm{CDCl}_{3}\right) \delta 131.7$, $128.31,127.6,124.2,90.3,84.7,80.8,68.4,28.7$, 28.5, 19.5, 18.5; IR $\left(\mathrm{cm}^{-1}\right) 3299,2936,2858,2236$, 2116, 1598, 1489; HRMS (ES+, $\left.\mathrm{CoCl}_{2}\right)$ for $\mathrm{C}_{20} \mathrm{H}_{20} \mathrm{OCoCl}$ cald: 304.0416 , found: 304.0429 .

Oil. ${ }^{1} \mathrm{H}$ NMR (400 MHz, $\left.\mathrm{CDCl}_{3}\right) \delta 7.32(\mathrm{~d}, J=8.7$ $\mathrm{Hz}, 2 \mathrm{H}), 6.81(\mathrm{~d}, J=8.7 \mathrm{~Hz}, 2 \mathrm{H}), 3.80(\mathrm{~s}, 3 \mathrm{H}), 2.40$ $(\mathrm{t}, J=7.0 \mathrm{~Hz}, 2 \mathrm{H}), 2.21(\mathrm{td}, J=6.9,2.5 \mathrm{~Hz}, 2 \mathrm{H})$, $1.95(\mathrm{t}, J=2.5 \mathrm{~Hz}, 1 \mathrm{H}), 1.55-1.63(\mathrm{~m}, 4 \mathrm{H}), 1.46(\mathrm{~m}$, $4 \mathrm{H}) .{ }^{13} \mathrm{C}$ NMR $\left(100 \mathrm{MHz}, \mathrm{CDCl}_{3}\right) \delta 156.1,132.0$, $116.3,113.9,88.7,84.7,80.4,76.8,55.3,28.8,28.4$, 19.4, 18.4; IR ( $\left.\mathrm{cm}^{-1}\right)$ 3295, 2934, 2858, 1606, 1508 ; HRMS (ES+, $\left.\mathrm{CoCl}_{2}\right)$ for $\mathrm{C}_{20} \mathrm{H}_{20} \mathrm{OCoCl}$ cald: 334.0539, found: 334.0535 .

1. Katritzky, A. R.; Abdel-Fattah, A. A. A.; Wang, M. J. Org. Chem. 2002, 67, 7526.

2. Denmark, S. E.; Tymonko, S. A. J. Org. Chem. 2003, 68, 9151.

3. Chapdelaine, M.; Warwick, P.; Shaw, A. J. Org. Chem. 1989, 54, 1218. 
4. Singh, R.; Just, G. J. Org. Chem. 1989, 54, 4453.

5. King, A. O.; Negishi, E.; Villani, F. J. Jr.; Silveira, A. Jr. J. Org. Chem. 1978, 43, 358.

6 Commercially available.

7 Sakai, N.; Annaka, K.; Konakahara, T. Org. Lett. 2004, 6, 1527.

8 Karpov, A. S.; Rominger, F.; Müller, T. J. J. J. Org. Chem. 2003, 68, 1503. 
n

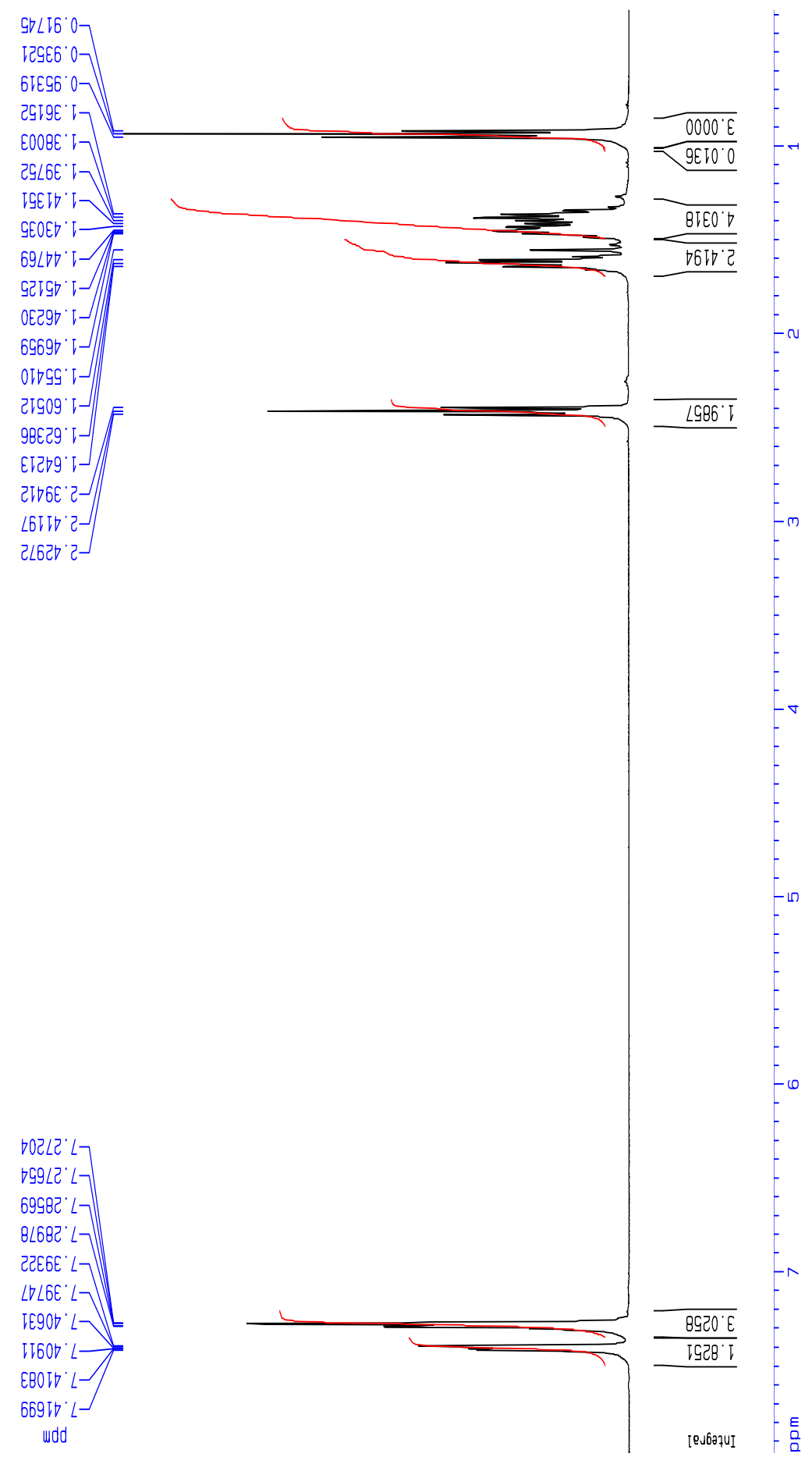




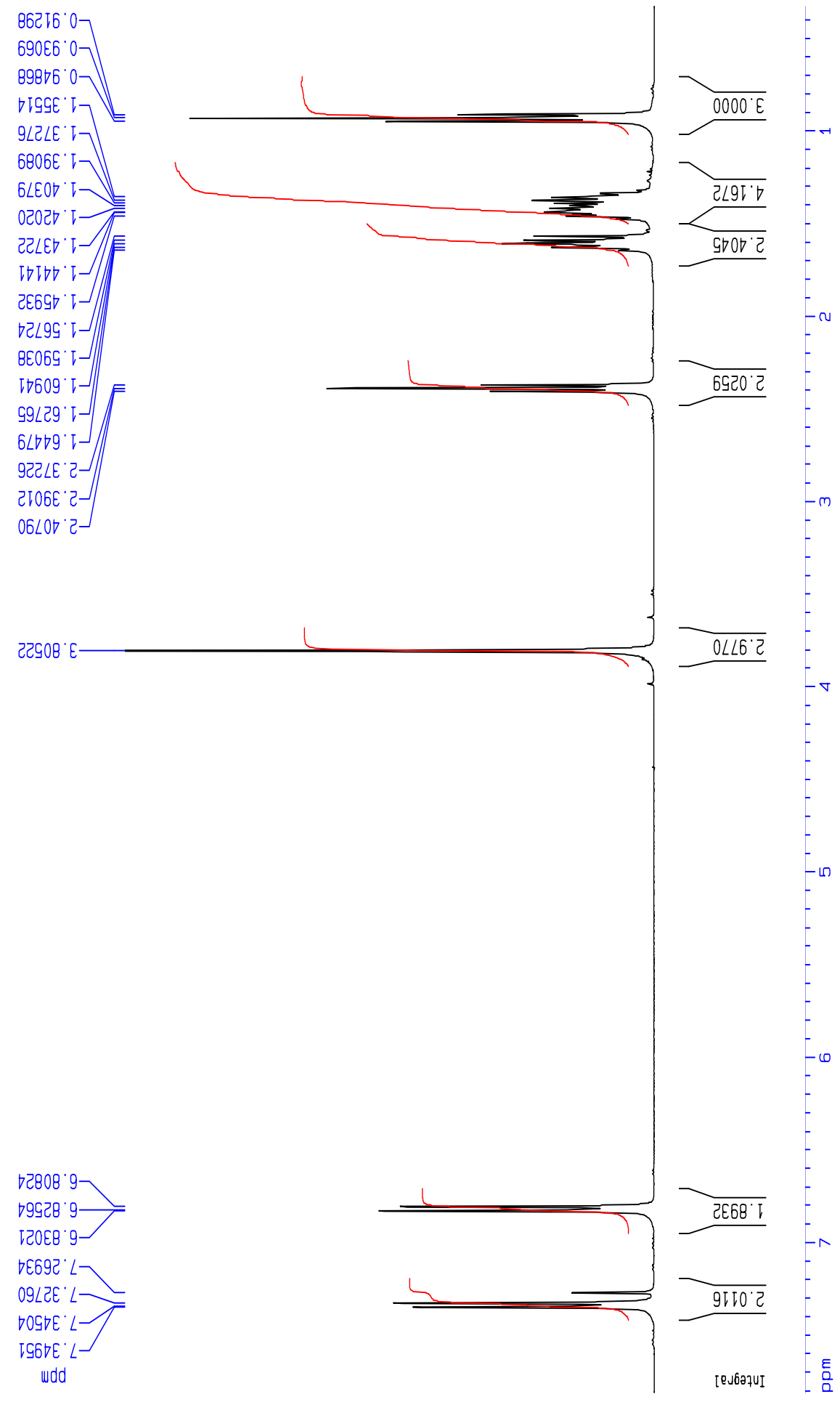




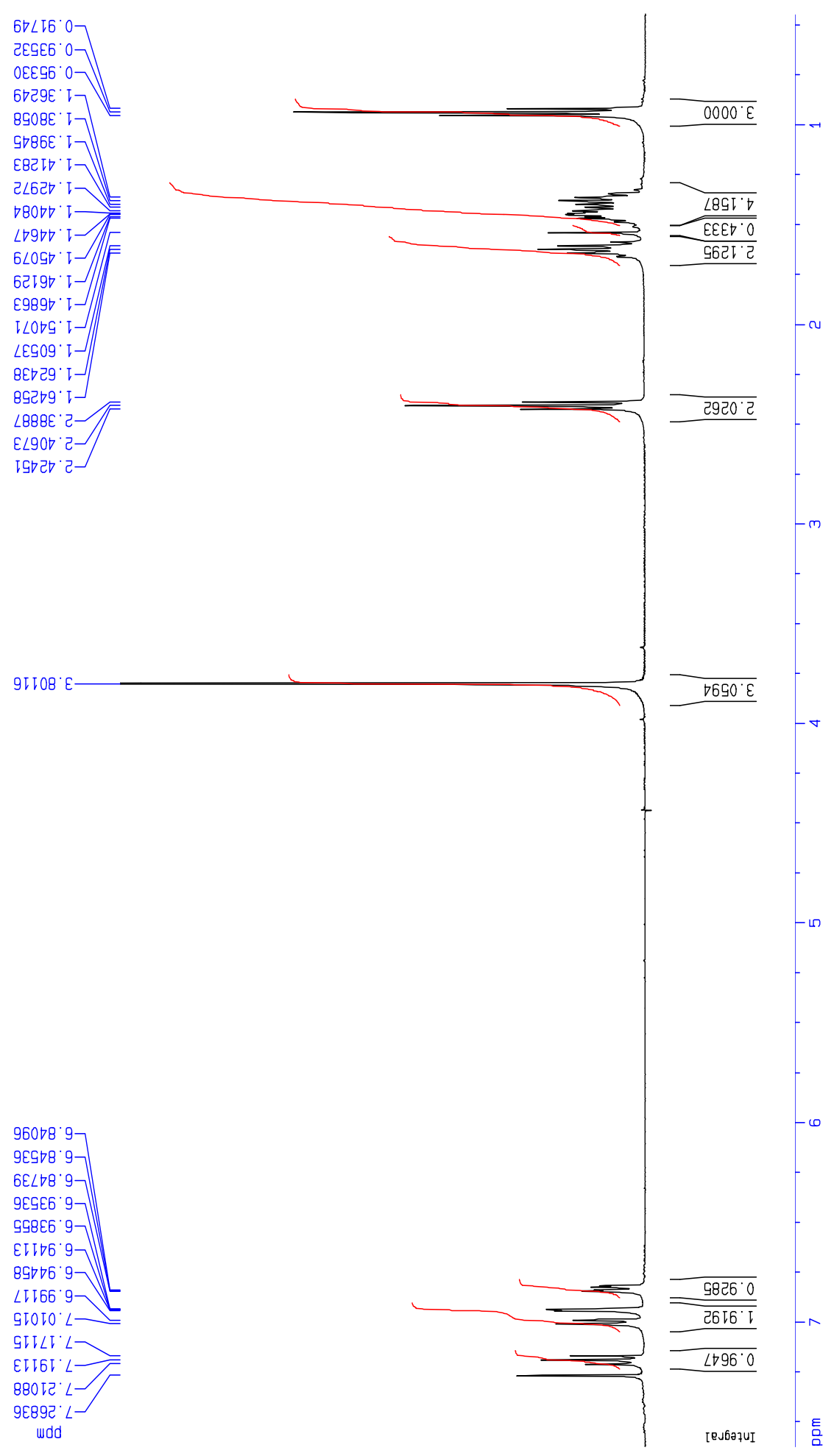




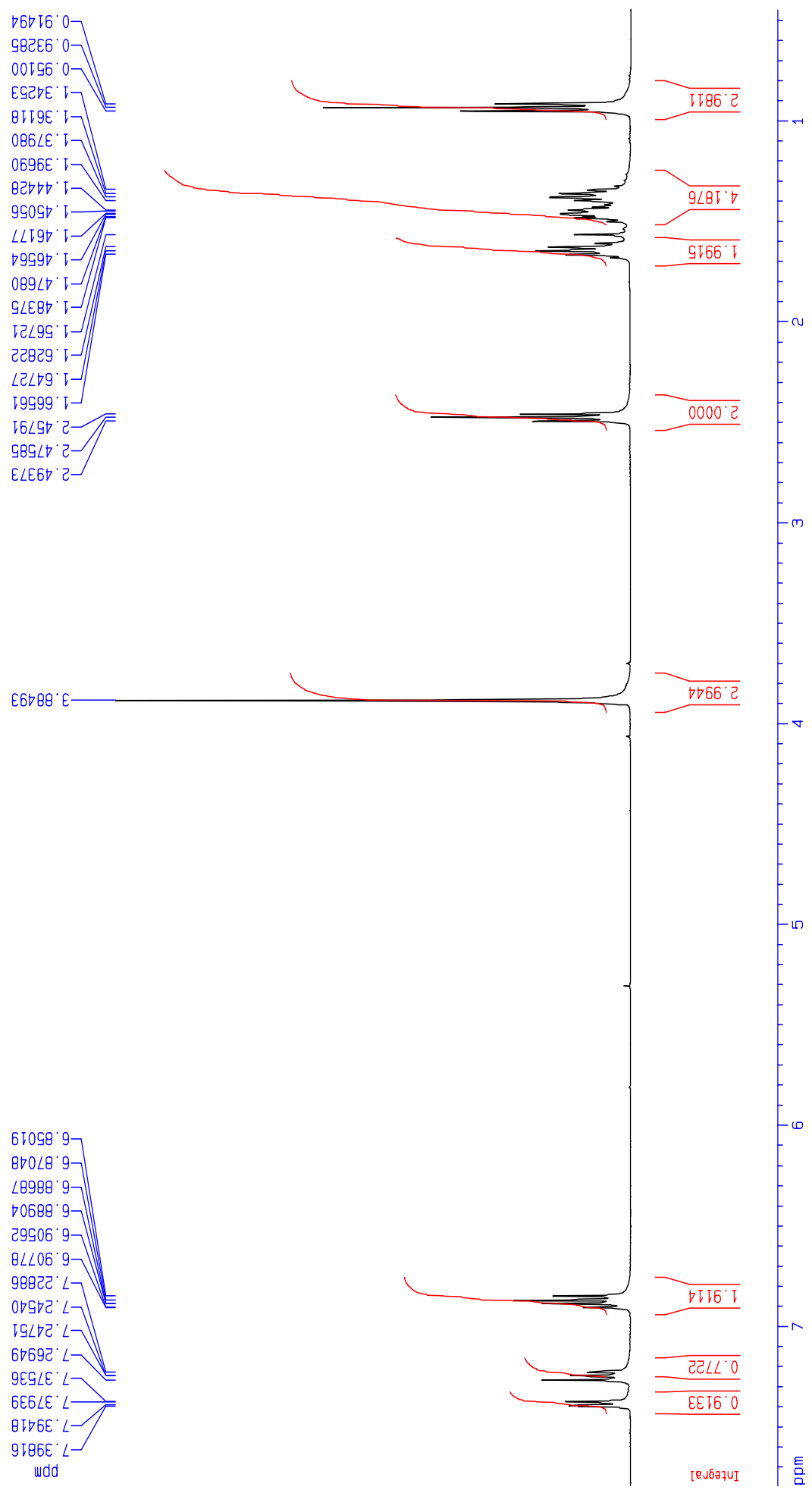




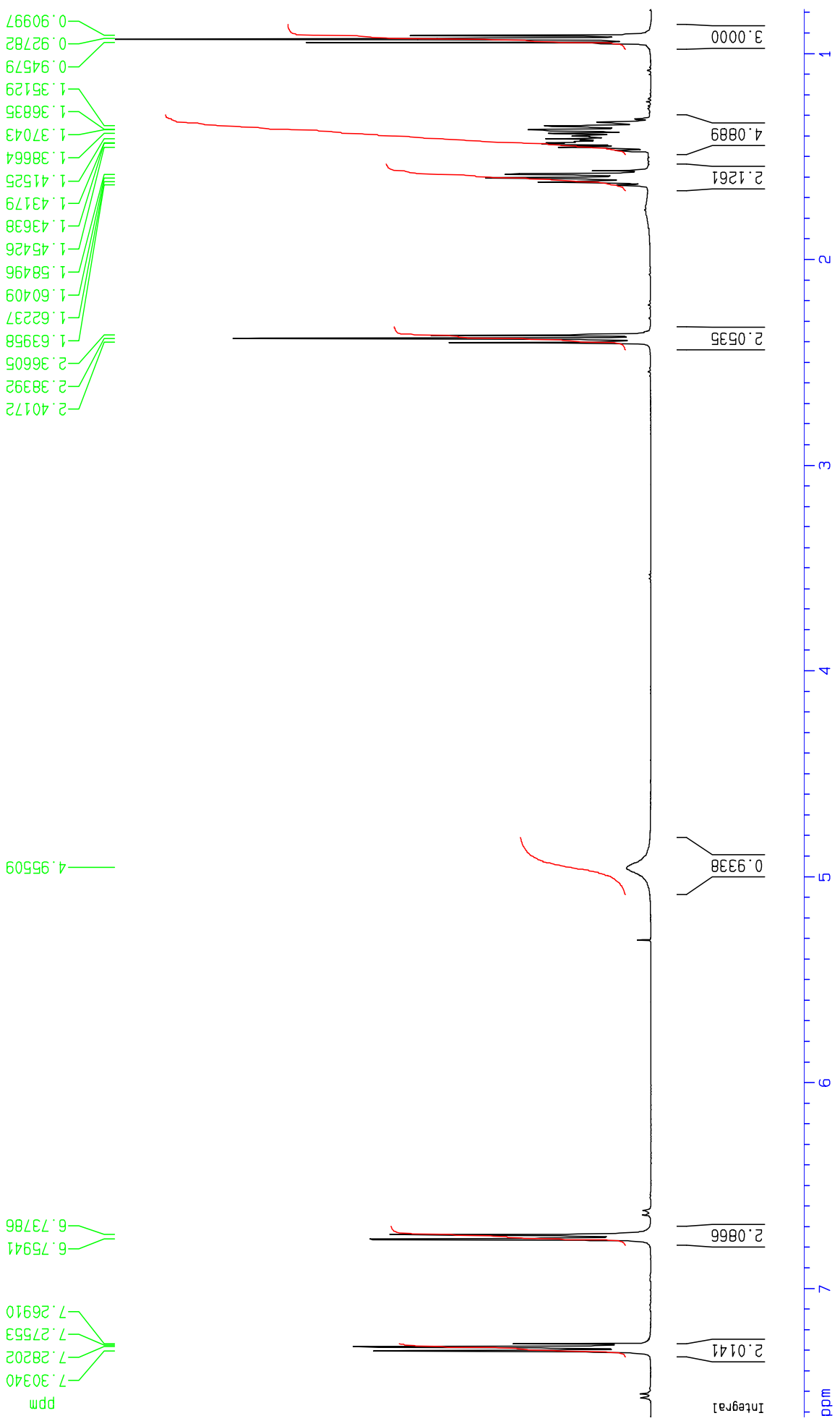




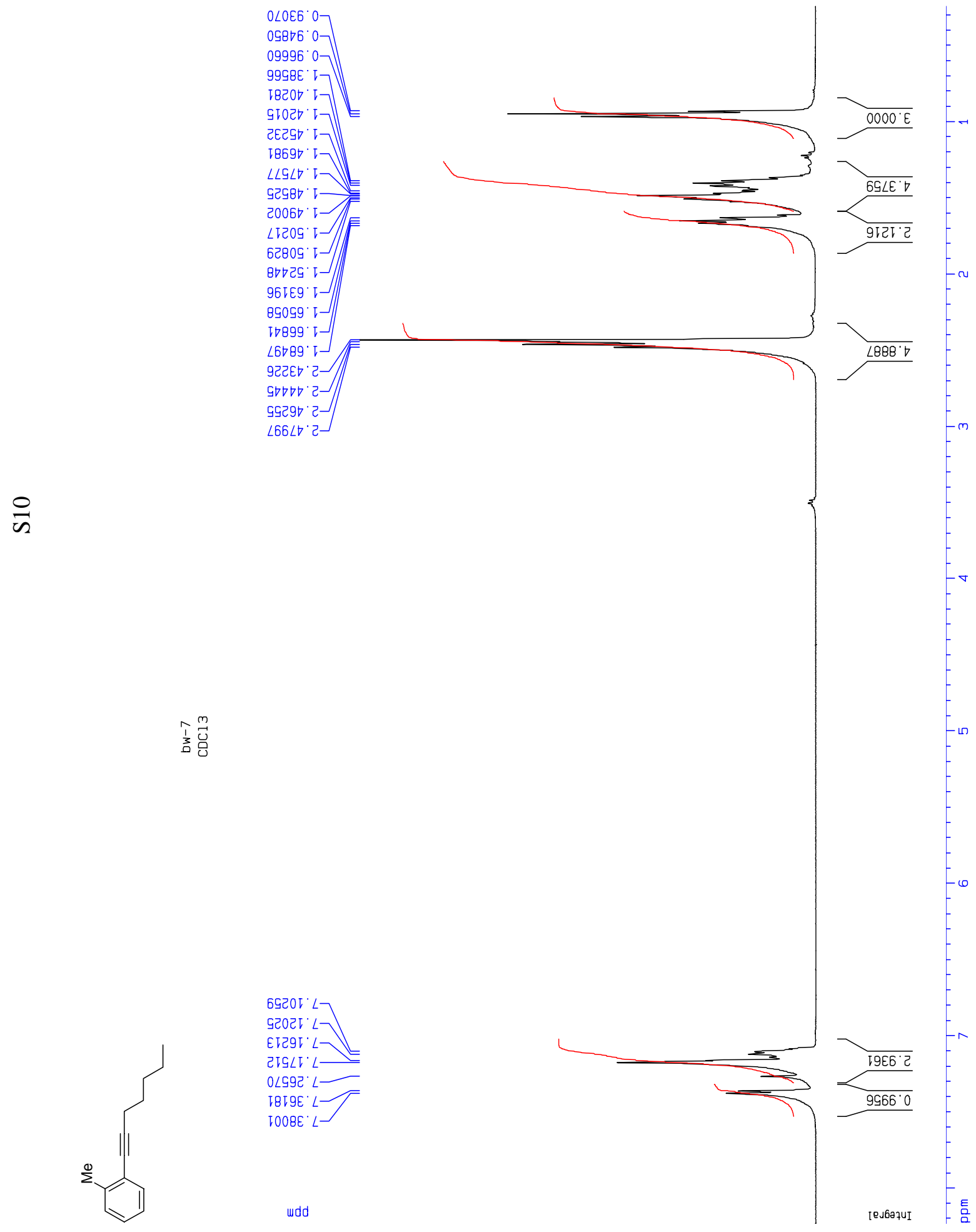




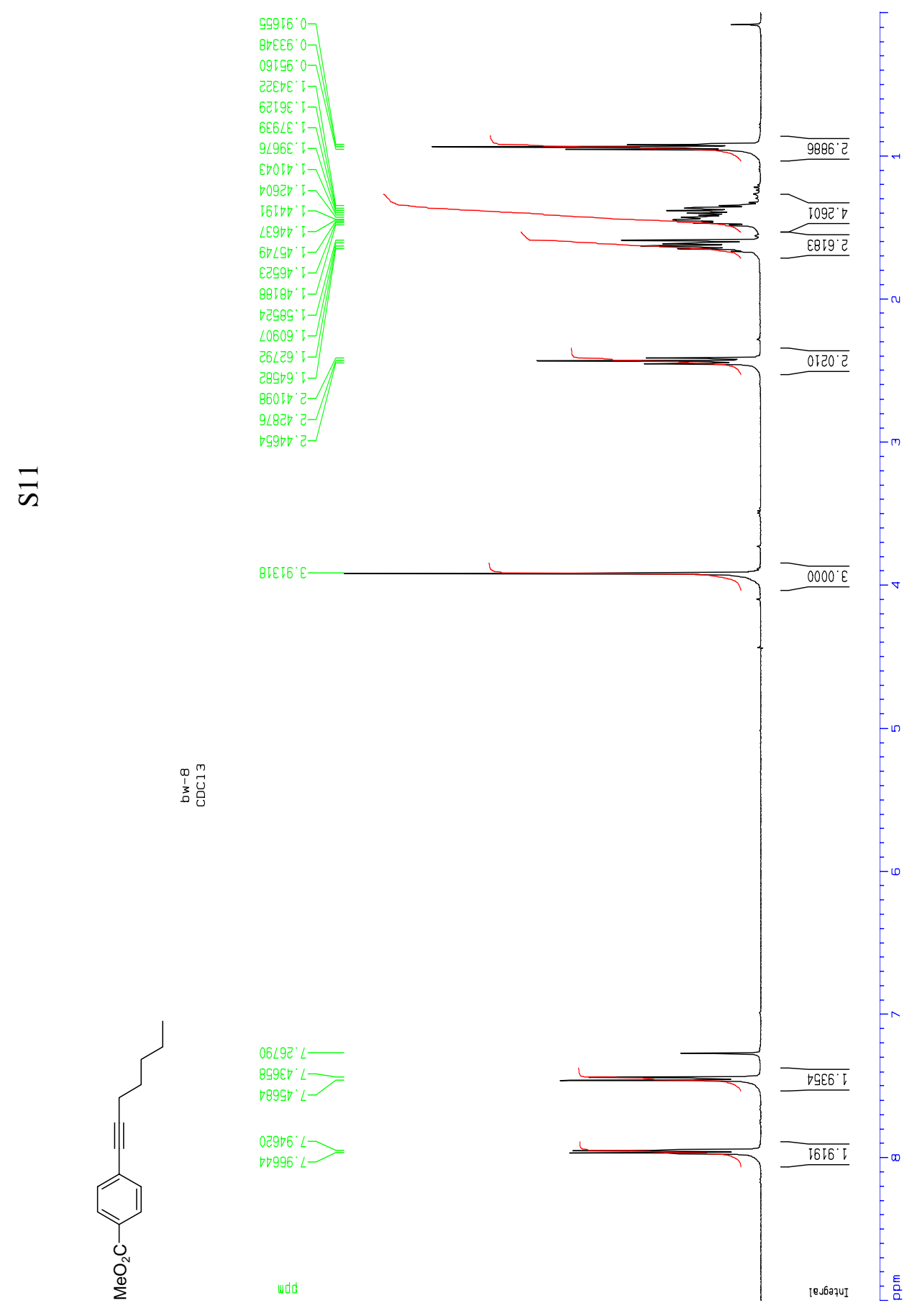




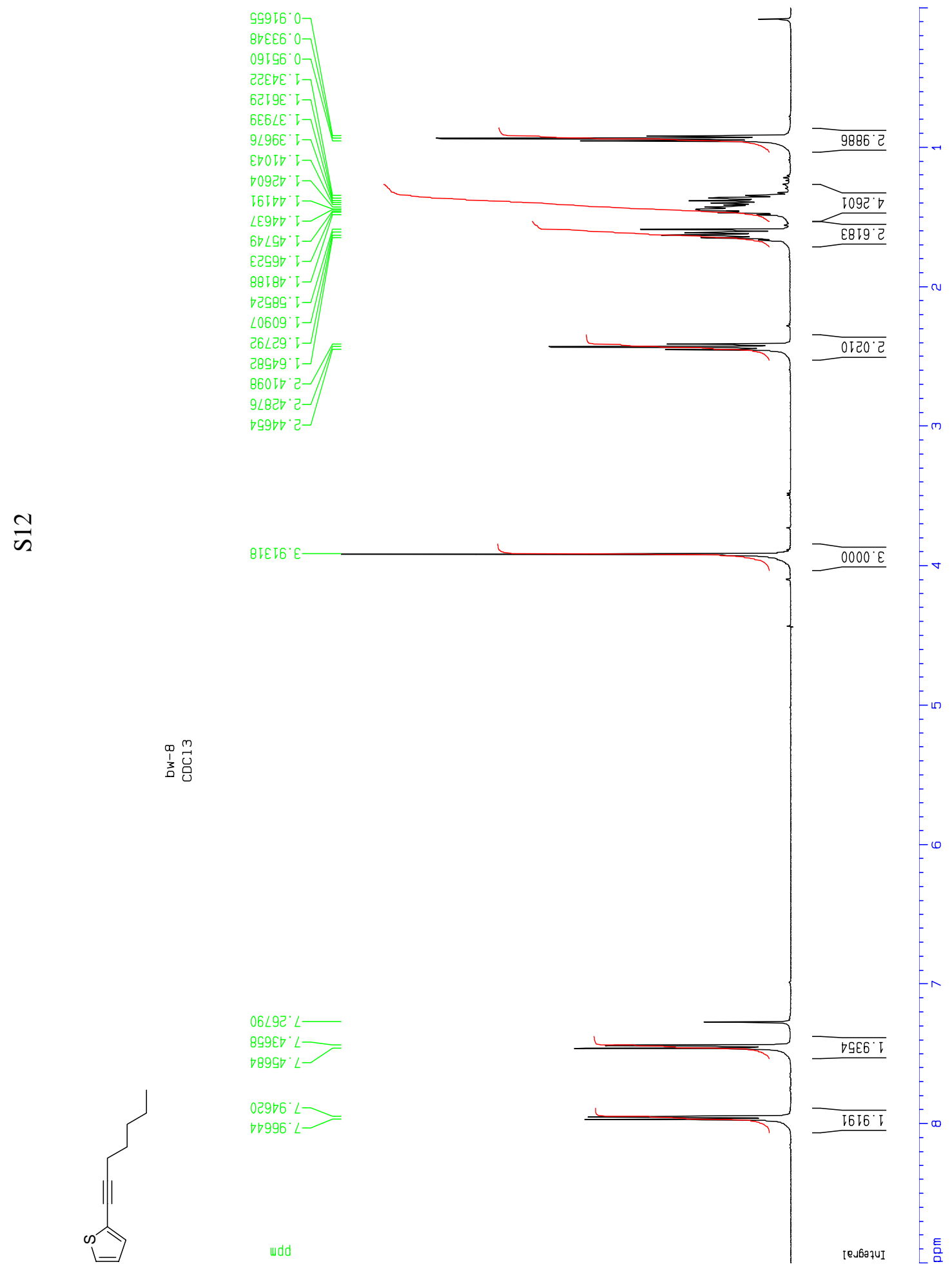



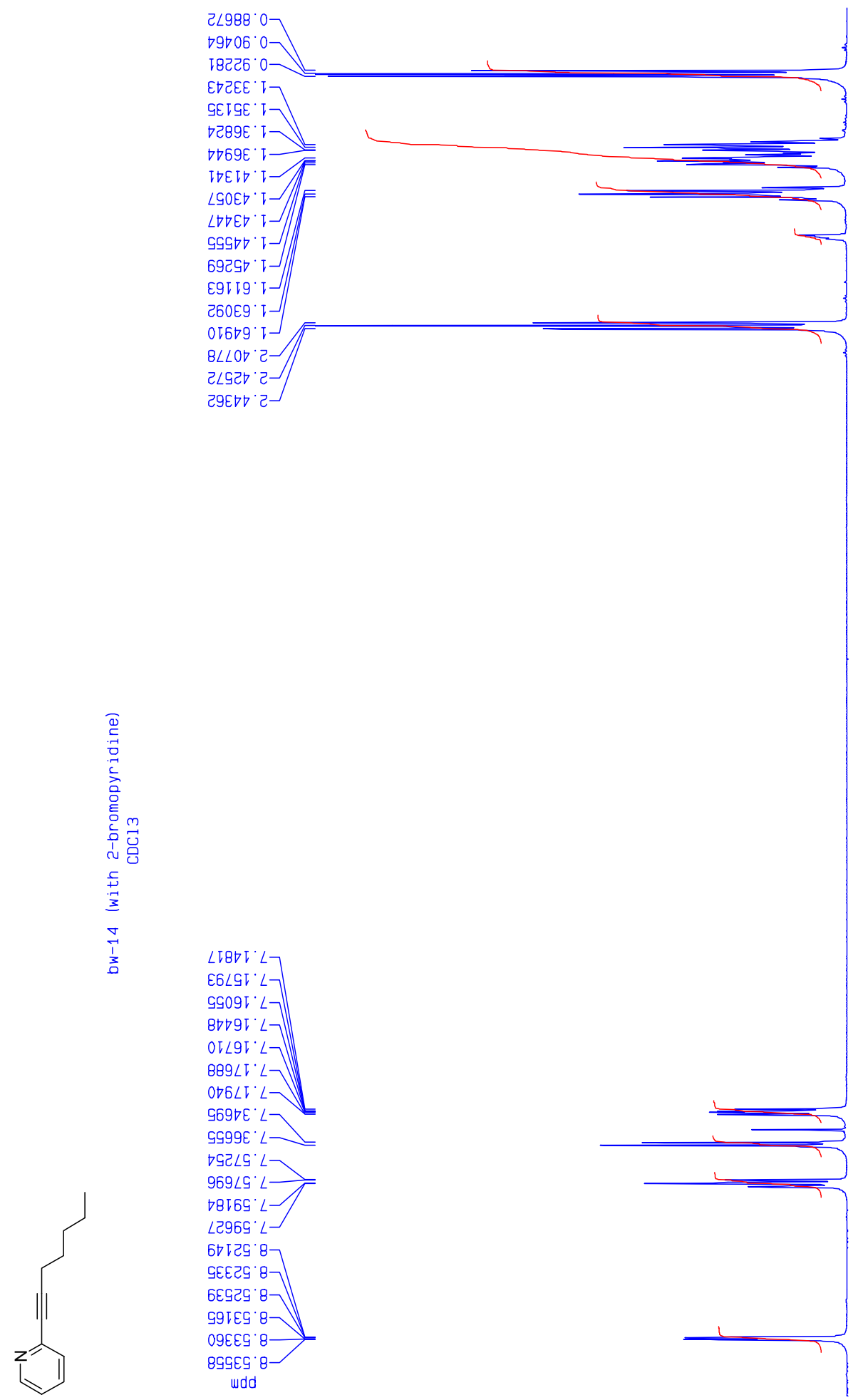

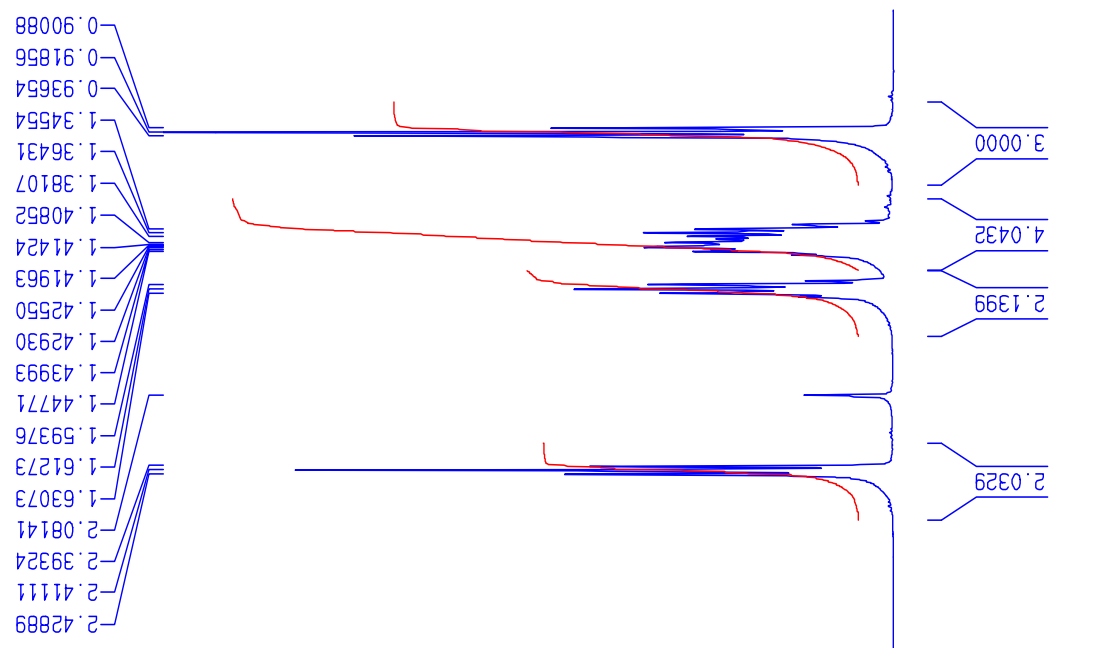

$\frac{\Xi}{n}$
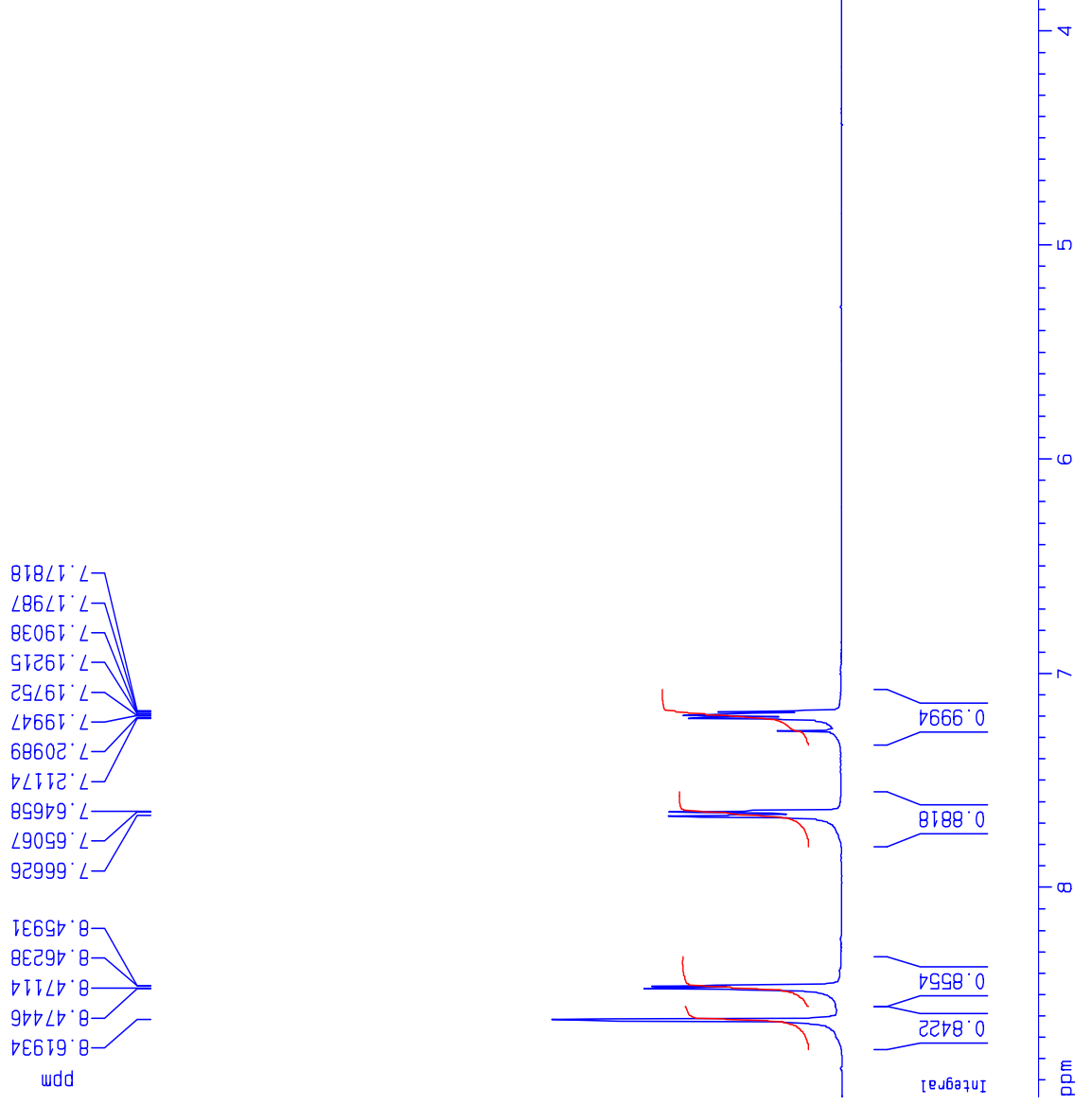


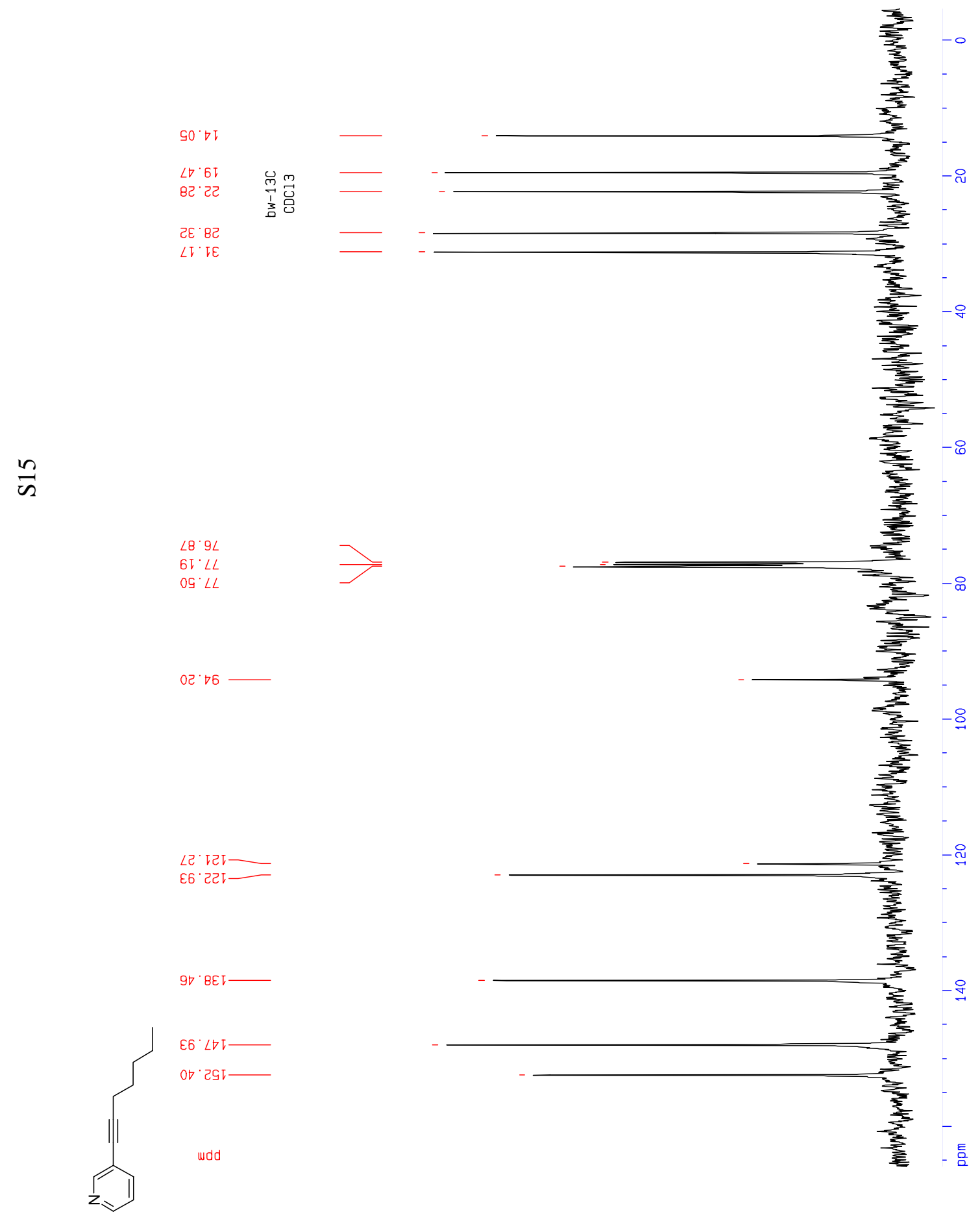




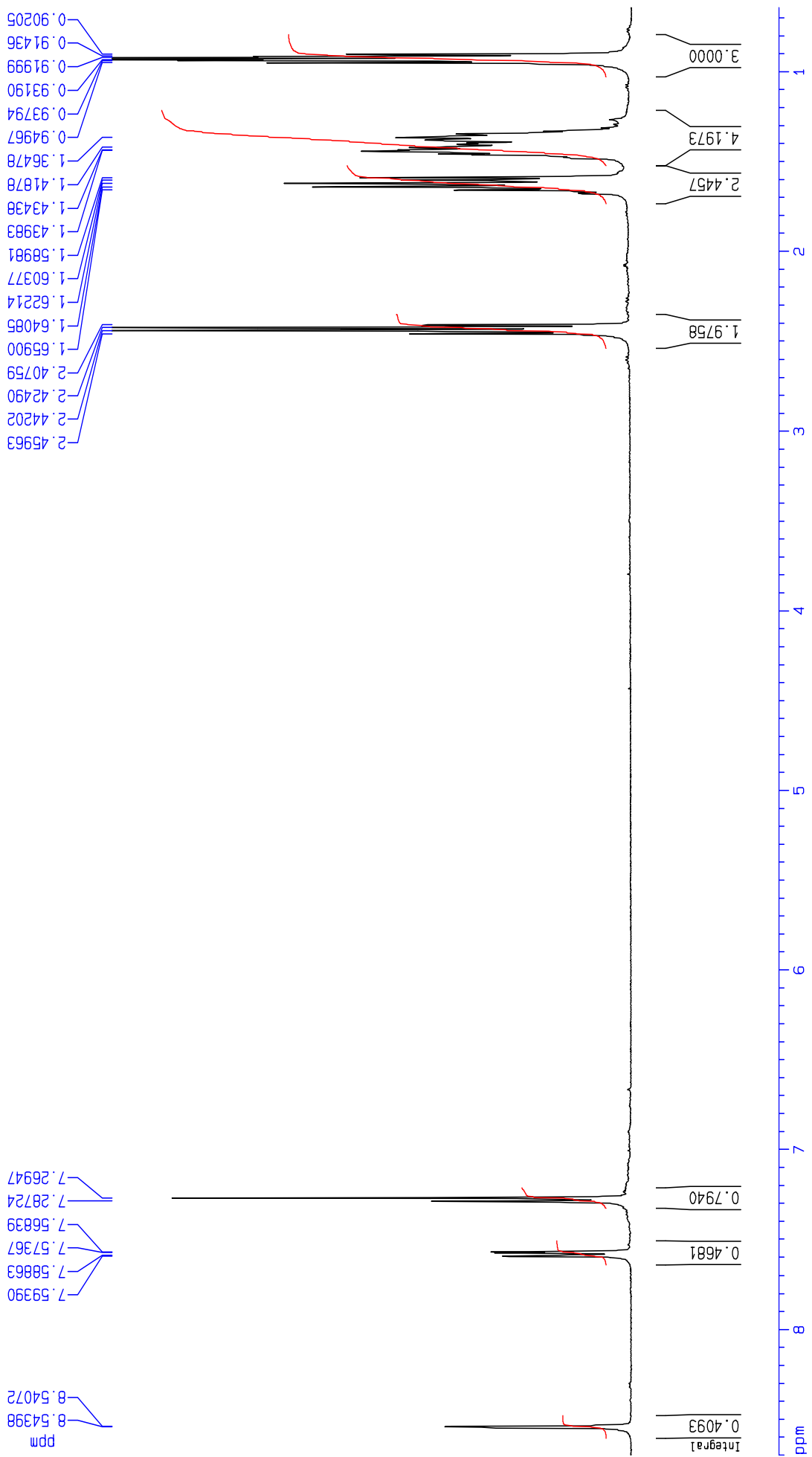



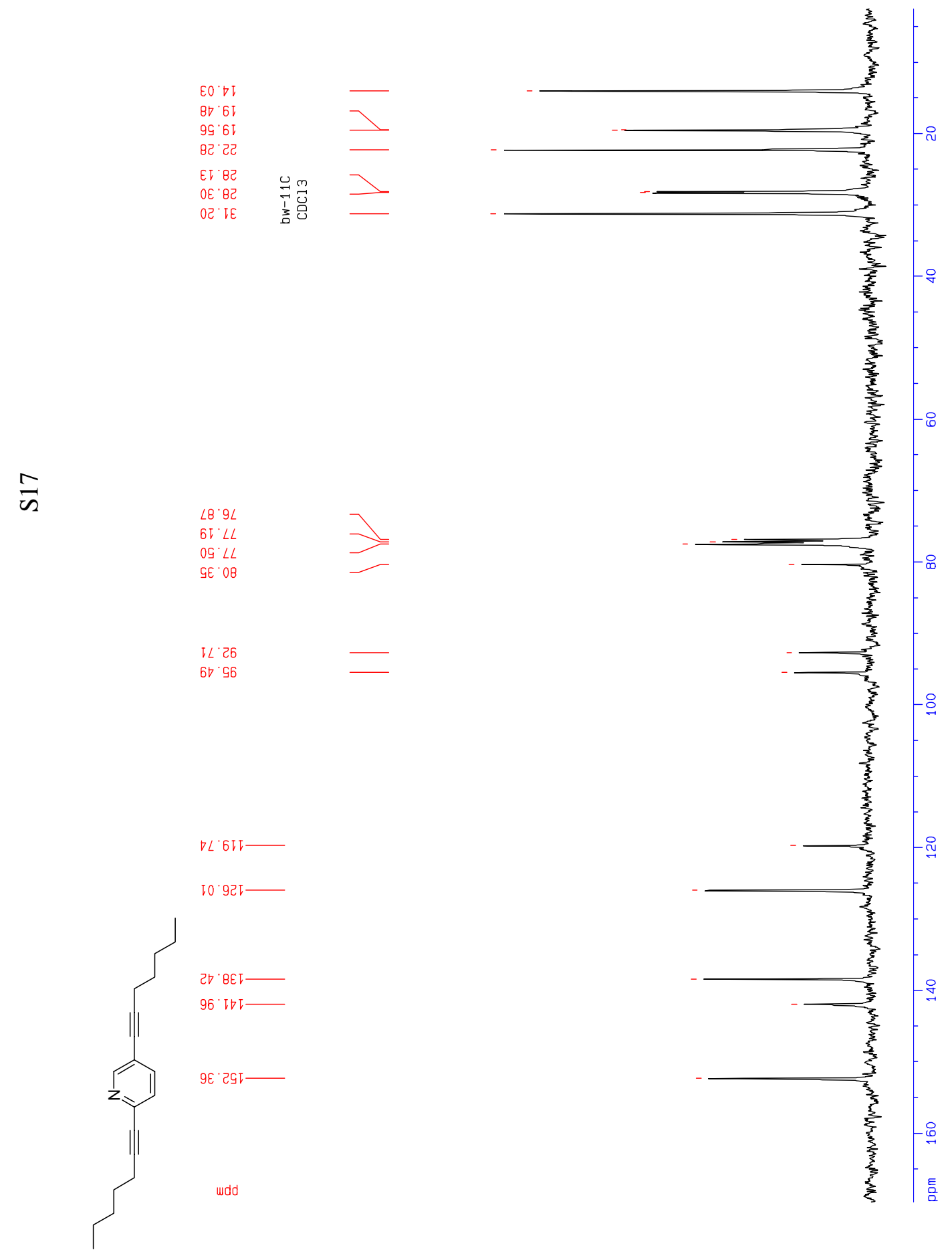


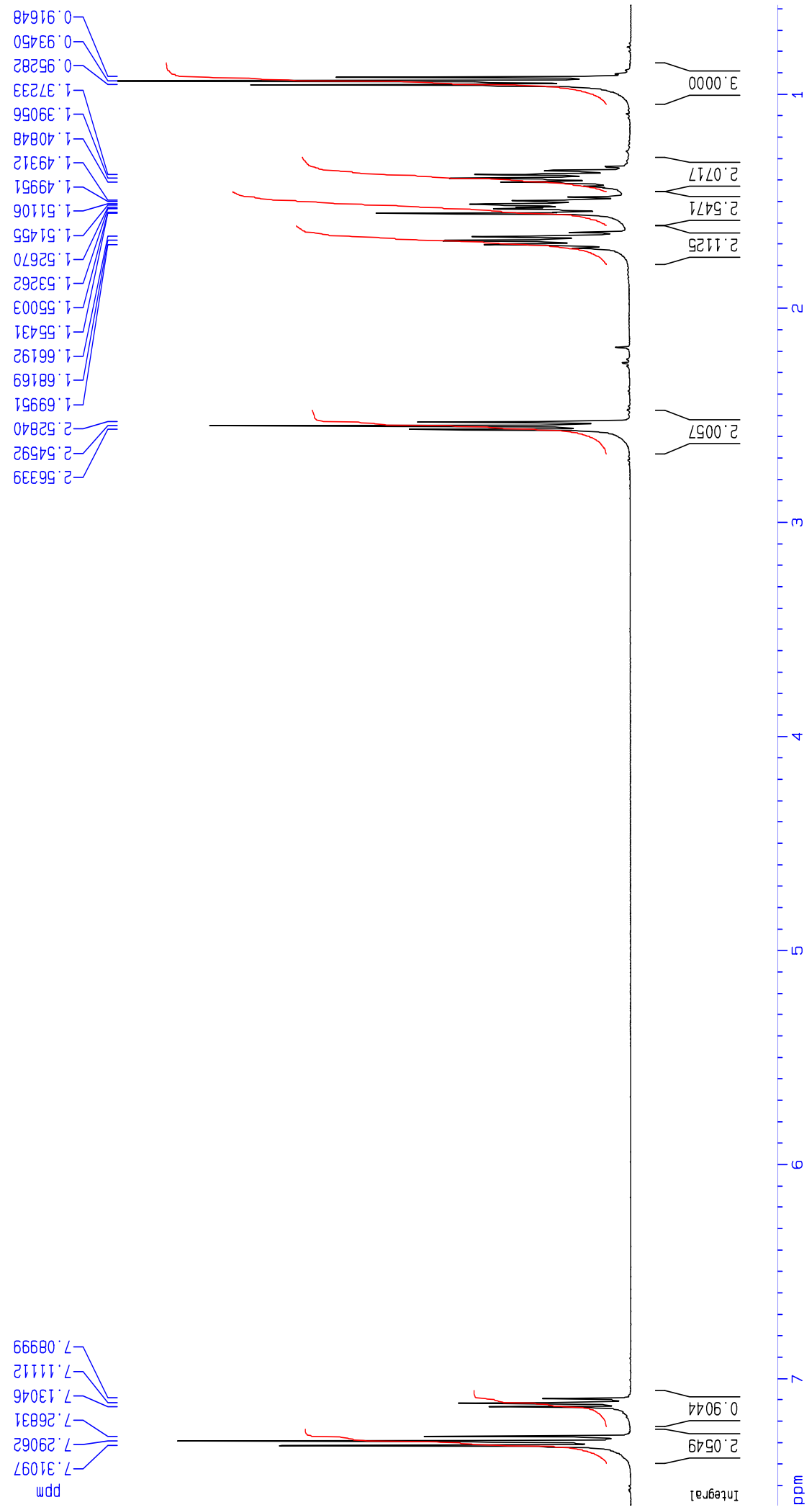



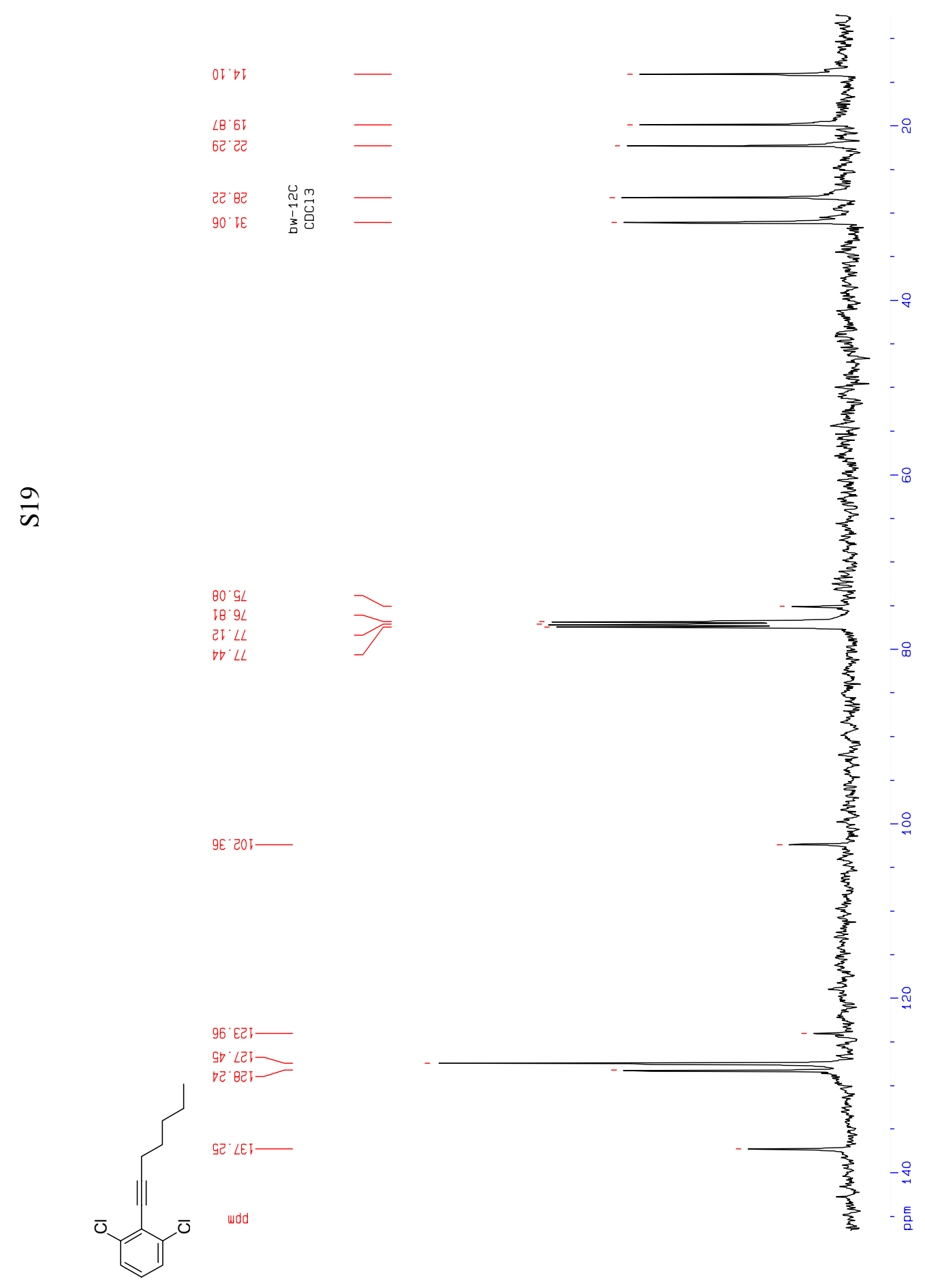
ฉิ

咢总

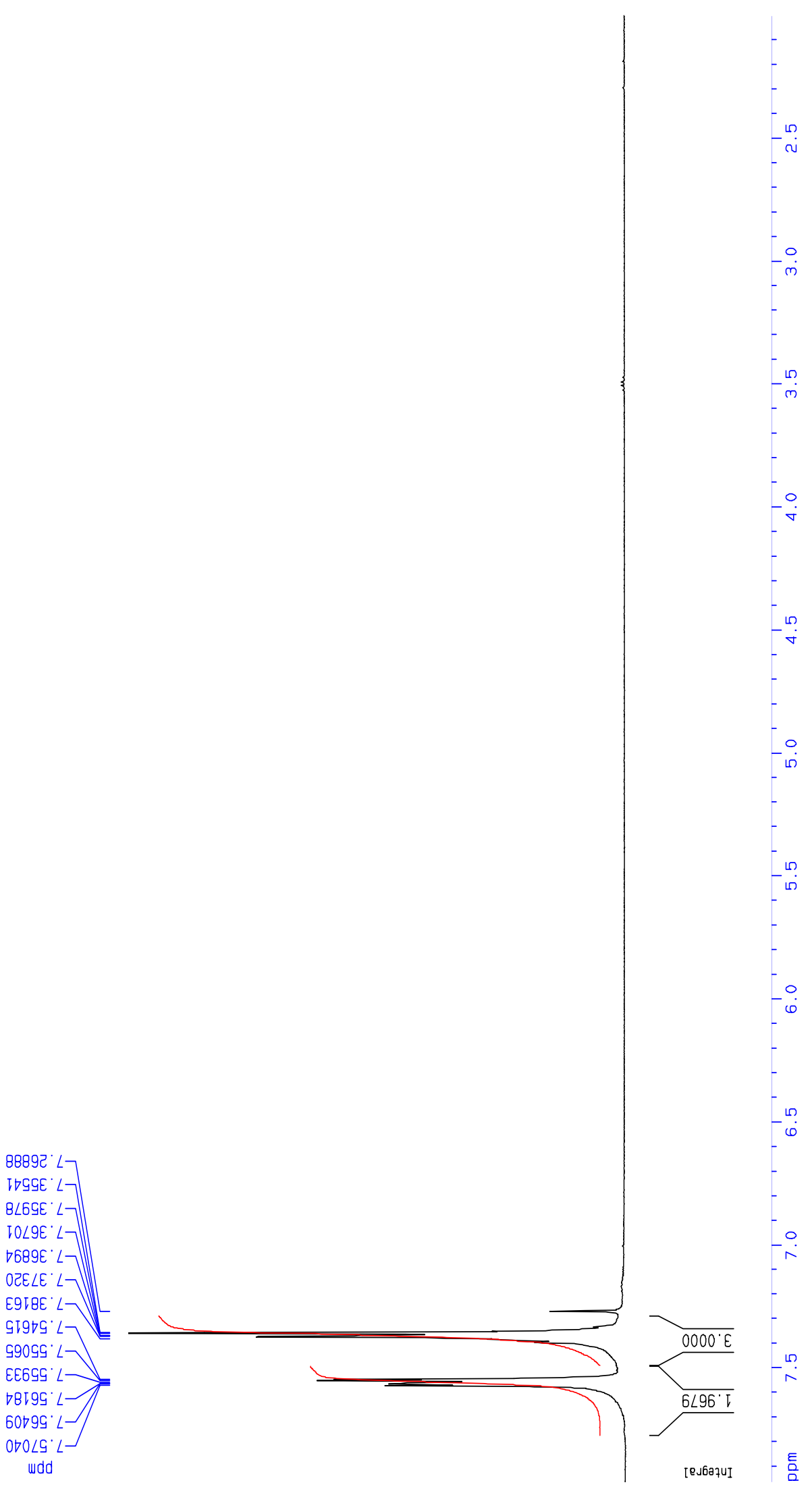


$\bar{N}$

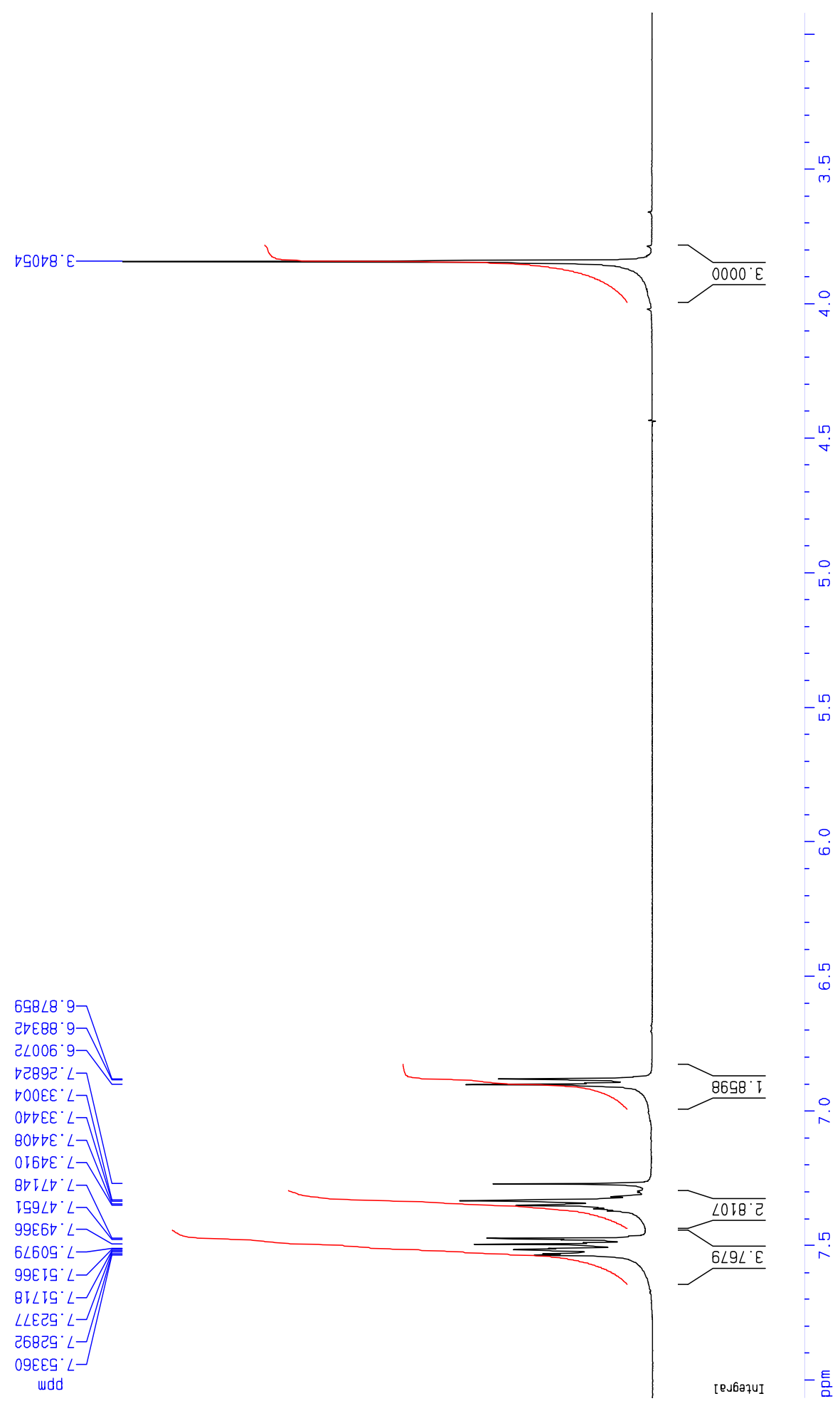




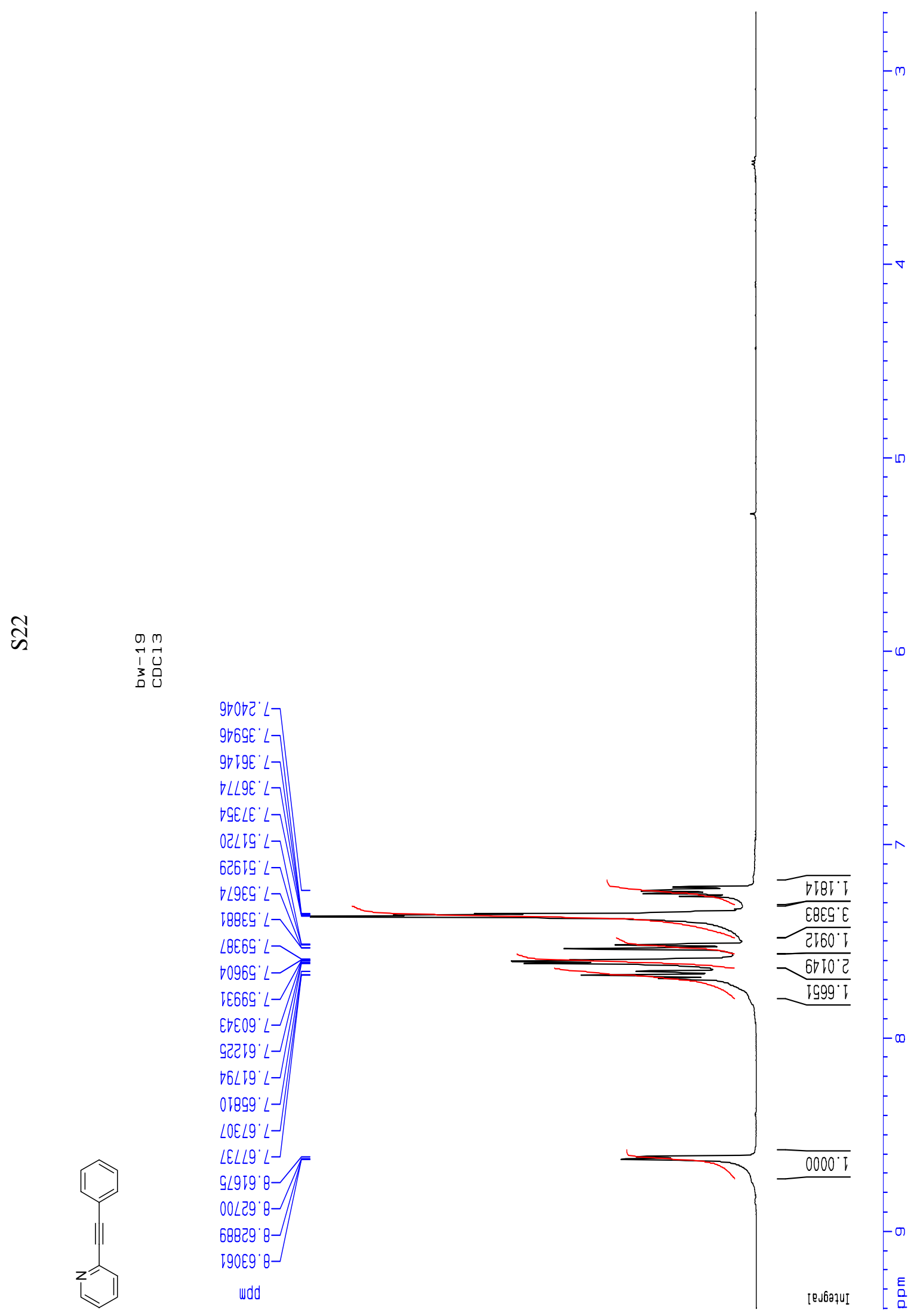




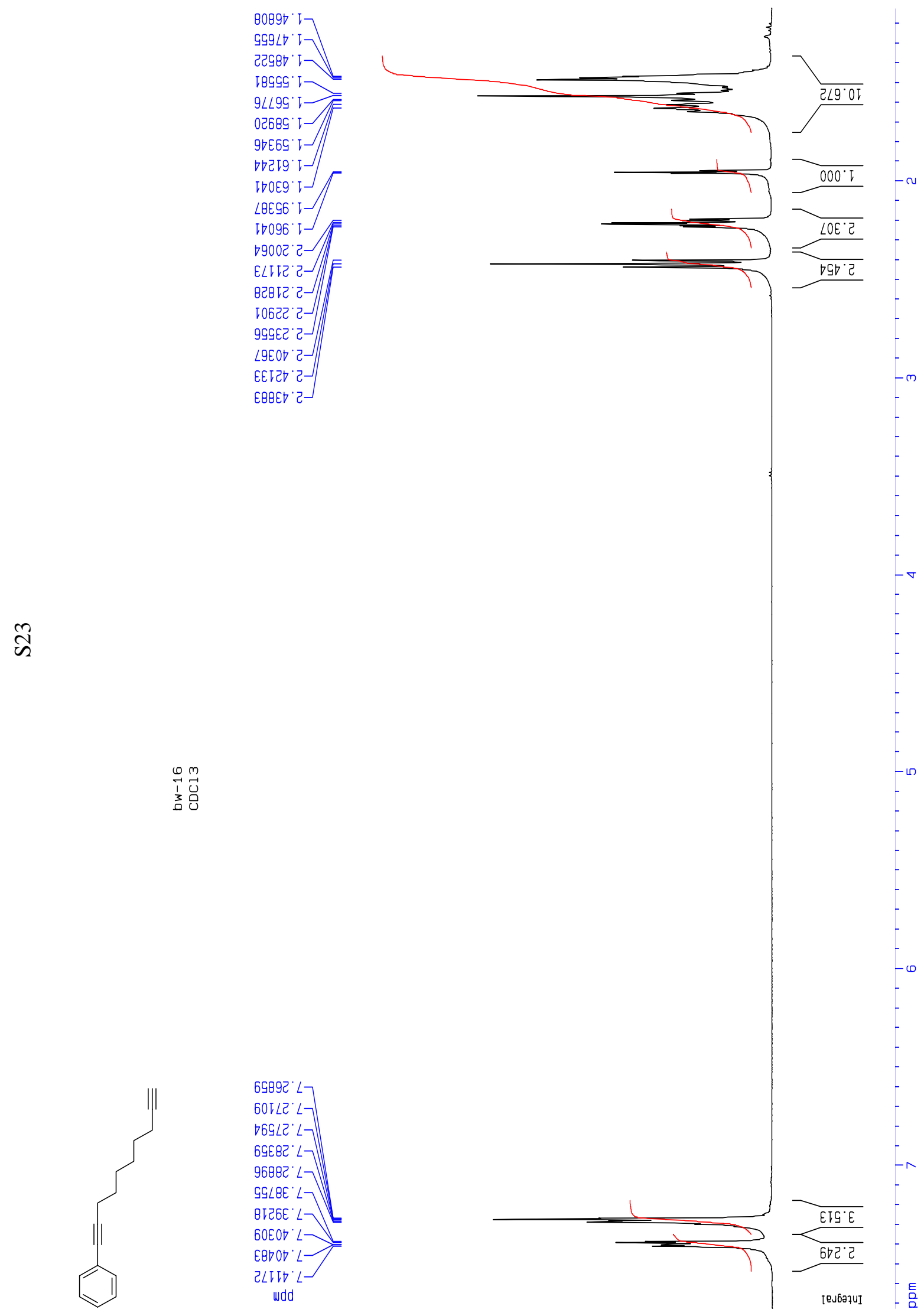




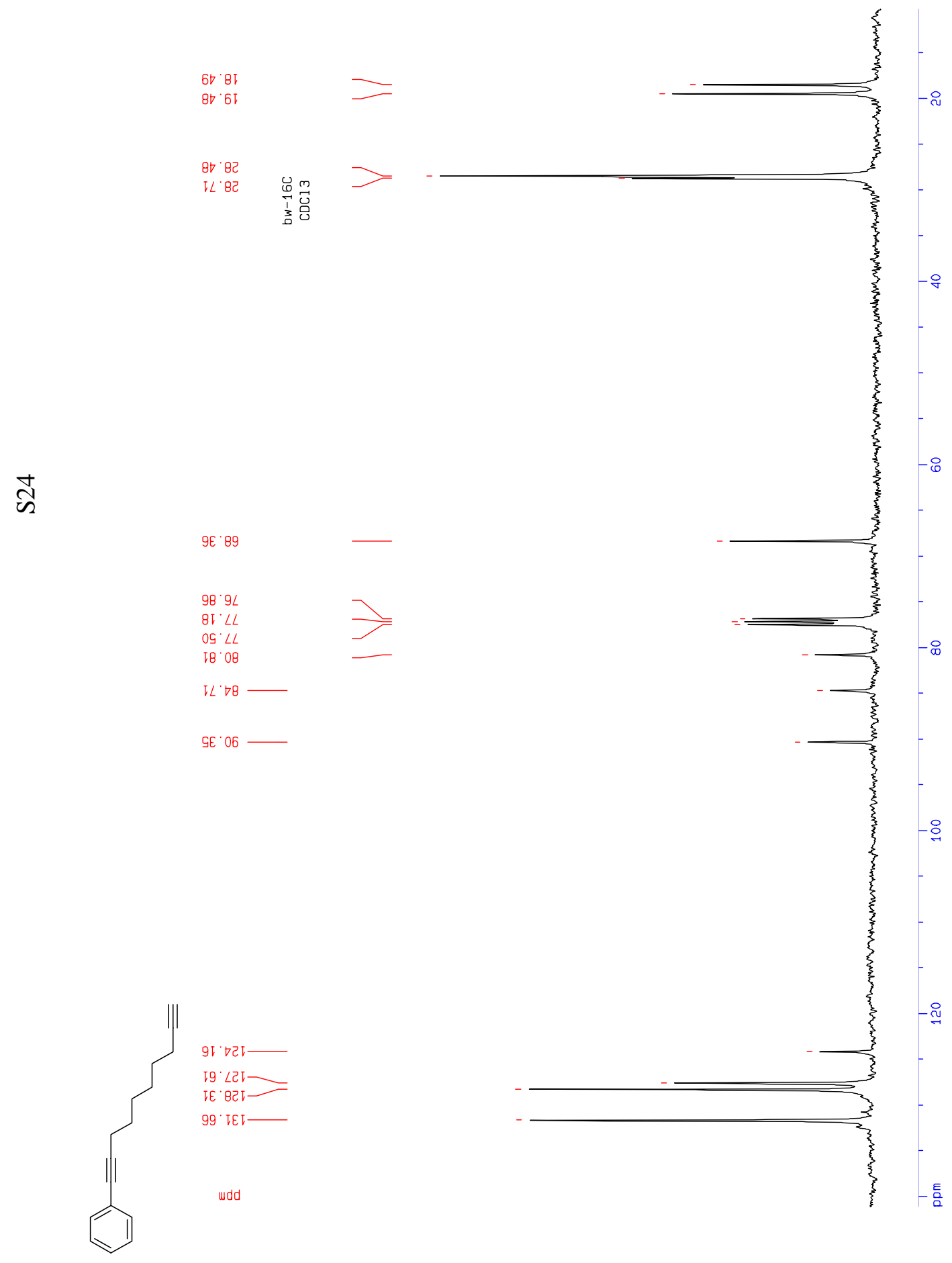




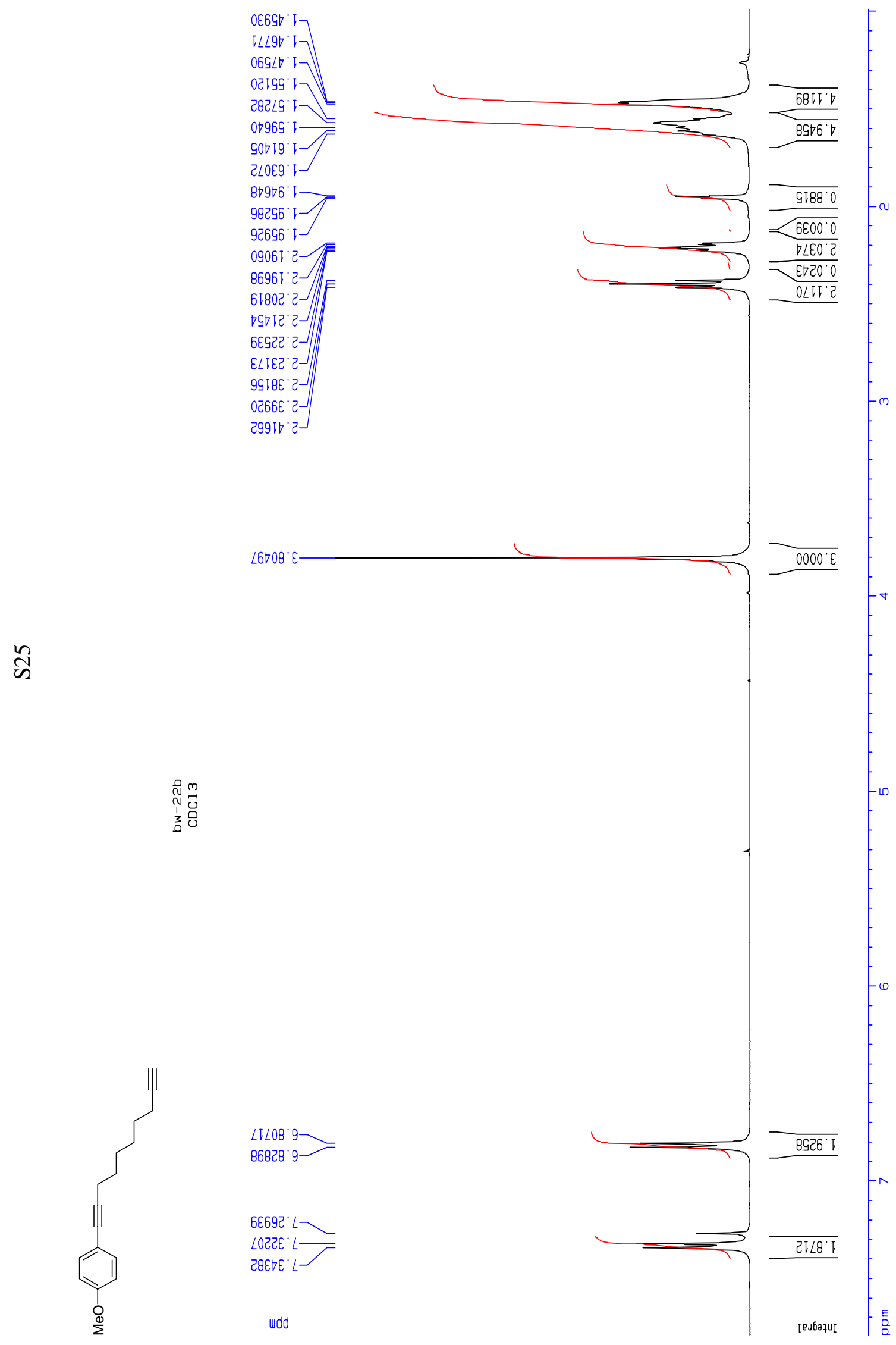



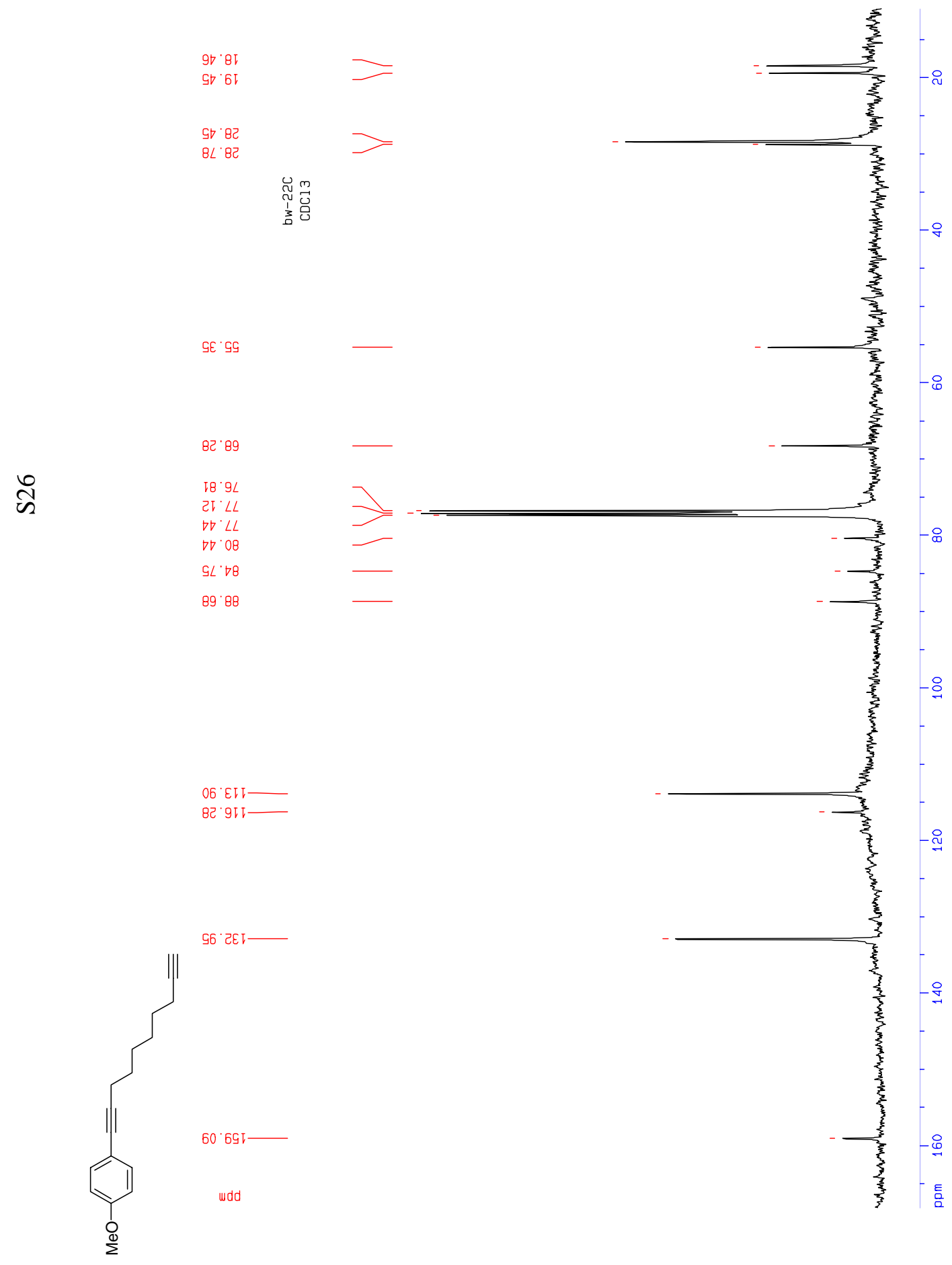


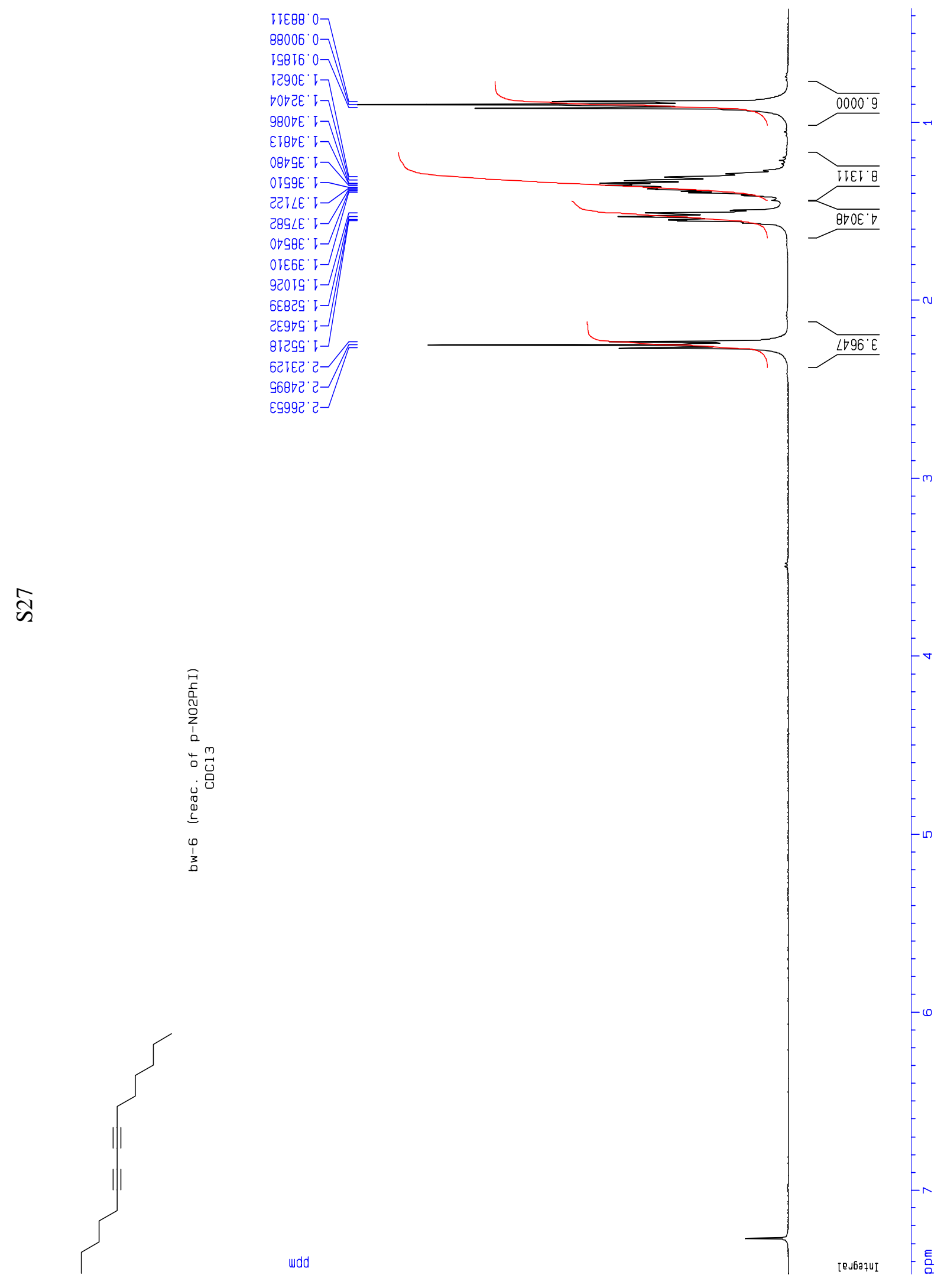




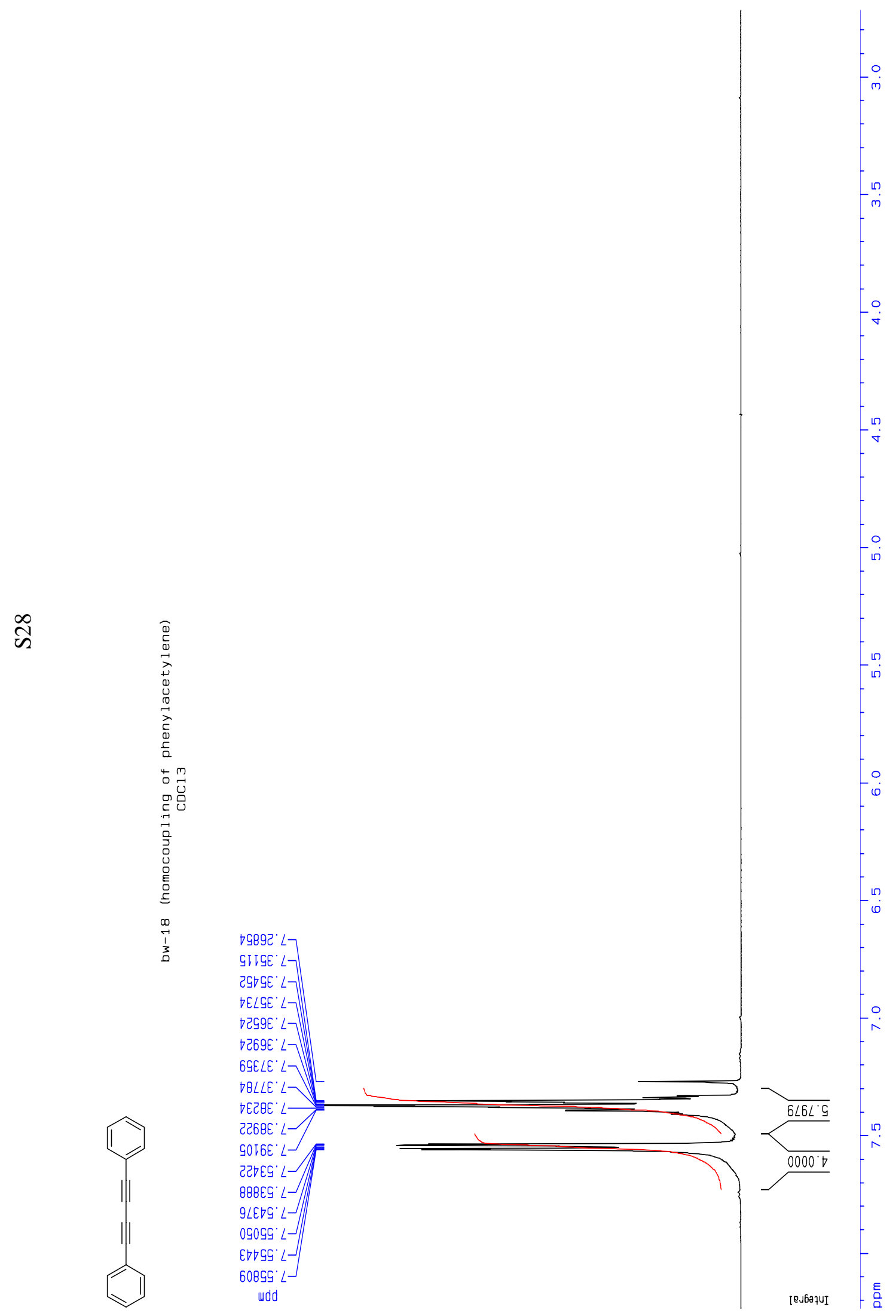

